DOI 10.4171/JEMS/244

Dave Anderson · Stephen Griffeth · Ezra Miller

\title{
Positivity and Kleiman transversality in equivariant $K$-theory of homogeneous spaces
}

Received December 23, 2008 and in revised form February 15, 2010

\begin{abstract}
We prove the conjectures of Graham-Kumar [GrKu08] and Griffeth-Ram [GrRa04] concerning the alternation of signs in the structure constants for torus-equivariant $K$-theory of generalized flag varieties $G / P$. These results are immediate consequences of an equivariant homological Kleiman transversality principle for the Borel mixing spaces of homogeneous spaces, and their subvarieties, under a natural group action with finitely many orbits. The computation of the coefficients in the expansion of the equivariant $K$-class of a subvariety in terms of Schubert classes is reduced to an Euler characteristic using the homological transversality theorem for nontransitive group actions due to S. Sierra. A vanishing theorem, when the subvariety has rational singularities, shows that the Euler characteristic is a sum of at most one term-the top one-with a well-defined sign. The vanishing is proved by suitably modifying a geometric argument due to M. Brion in ordinary $K$-theory that brings Kawamata-Viehweg vanishing to bear.
\end{abstract}

Keywords. Flag variety, equivariant $K$-theory, Kleiman transversality, homological transversality, Schubert variety, Borel mixing space, rational singularities, Bott-Samelson resolution

\section{Introduction}

The structure constants of cohomology rings of homogeneous spaces tend to exhibit positivity properties. Combinatorics often enters, through attempts to interpret positive quantities as enumerators, but it is by geometric means that the positivity is often first—or most easily - verified (a notable exception being the order of events relating [Buc02] and [Bri02]). In the typical setup, going back to Ehresmann [Ehr34], the cohomology ring in question possesses an additive basis of classes carried by algebraic subvarieties. Using the transitive group action, as pioneered by Kleiman [Kle74], these Schubert subvarieties can be translated generically; subsequently intersecting them yields cycles whose multiplicities are positive by virtue of being algebraic. These multiplicities are the structure constants, which are hence positive.

D. Anderson: Department of Mathematics, University of Washington, Seattle, WA 98195, USA; e-mail: dandersn@math.washington.edu

S. Griffeth: School of Mathematics, University of Edinburgh, Edinburgh EH9 3JZ, UK; e-mail: S.Griffeth@ed.ac.uk

E. Miller: Mathematics Department, Duke University, Durham, NC 27708, USA;

e-mail: ezra@math.duke.edu 
That positivity extends beyond ordinary cohomology has recently been demonstrated in two instances. Graham generalized it to torus-equivariant cohomology of the homogeneous spaces $G / P[\mathrm{Gra01}]$, confirming and extending conjectures of Billey and Peterson (cf. [Gra01, Section 4]). At about the same time, Brion proved it for ordinary $K$-theory [Bri02], after it had been conjectured by Buch [Buc02]. The very notion of positivity depends on the context, of course. In $K$-theory, positivity means sign alternation: if the dimension of a subvariety differs from the expected dimension by $i$ in a given intersection product, then the sign of the coefficient on its class is $(-1)^{i}$. For equivariant cohomology, the coefficients are polynomials; positivity means that, expressed as polynomials in the simple roots, the coefficients are nonnegative integers.

In view of the developments for ordinary $K$-theory and equivariant cohomology, it should come as no surprise that conjectures for equivariant $K$-theory, posed by GriffethRam [GrRa04] and Graham-Kumar [GrKu08], predict sign alternation in equivariant $K$ theory. These conjectures again make precise the notion of positivity for polynomials in terms of which the alternation is phrased. The aim of this paper is to derive these conjectures (Corollaries 5.1 5.3) from an appropriate generalization of Kleiman transversality (Theorem 4.2).

Several special cases of our main results have been proved over the last two decades. The first steps in this direction concerned multiplication by the class of a line bundle: positive "Pieri" formulas in equivariant $K$-theory were given by Fulton-Lascoux in type $A$ [FuLa94], and by Pittie-Ram [PiRa99] and Mathieu [Mat00] for general $G / B$. GriffethRam verified their conjecture for all rank 2 groups, and Graham-Kumar proved their conjecture for projective spaces, as well as Schubert expansions of opposite Schubert varieties in any $G / B$.

Ordinary Kleiman transversality concerns the movement of subvarieties of a homogeneous space $G / P$ into general position using the transitive group action. This has positivity consequences for nonequivariant cohomology theories, including ordinary $K$ theory of $G / P$, because translation preserves rational equivalence. Equivariantly, on the other hand, translation alters the classes of cycles. Our equivariant homological Kleiman transversality principle, Theorem 7.2 and its strong version for subvarieties with rational singularities, Theorem 4.2 therefore take place on a Borel mixing space of $G / P$-or, more precisely, a finite-dimensional approximation $\mathcal{X}$-whose ordinary (i.e., nonequivariant) homological invariants are the equivariant invariants of $G / P$.

Roughly speaking, Theorems 4.2 and 7.2 say that $\mathcal{X}$ has a large group action, large enough so that the general translate of the mixing space $\mathcal{Y}$ of a torus-stable subvariety $Y$ (or arbitrary coherent sheaf on $G / P$ ) is homologically transverse in $\mathcal{X}$ to the mixing spaces $\mathcal{X}^{w}$ of the opposite Schubert subvarieties $X^{w} \subseteq G / P$. Consequently, each coefficient in the Schubert basis expansion of the torus-equivariant $K$-class $\left[\mathcal{O}_{Y}\right]$ can be computed as the Euler characteristic of a certain "boundary" divisor on the intersection $\mathcal{Y} \cap \gamma \cdot \mathcal{X}_{J}^{w}$ of the mixing space $\mathcal{Y}$ with a general translate of another mixing space $\mathcal{X}_{J}^{w}$ (Theorem 7.2). To be more precise, mixing spaces are bundles over a product $\mathbb{P}$ of large projective spaces (Section 2, and $\mathcal{X}_{J}^{w}$ is the restriction to a certain product of projective subspaces, indexed by $J$, of the mixing space $\mathcal{X}^{w}$. The strong version in Theorem 4.2 says that when $Y$ has rational singularities, the Euler characteristic is an alternating sum of terms in which only the last can be nonzero. 
The action of the mixing group $\Gamma$, introduced in Section 6 is derived from the structure of the mixing space $\mathcal{X}$ as a bundle with fiber $G / P$ over $\mathbb{P}$. The base $\mathbb{P}$ has a transitive automorphism group. The fibers $G / P$ obviously also have transitive automorphism groups, but a priori these only guarantee automorphisms of $\mathcal{X}$ over open subsets of $\mathbb{P}$. Constructing global automorphisms depends on constructing sections of a group scheme related to $\mathcal{X}$. This, in turn, ultimately relies on a certain positivity hypothesis on the torus action (Section 2.4) that pervades all of our main results.

In view of the applications in Section 5, the statements of our main results, particularly Theorems 4.1 and 4.2 as derived from Theorems 7.2 and 10.4, contain always two flavors: one expands equivariant classes in terms of equivariant Schubert structure sheaves $\mathcal{O}_{w}=$ $\left[\mathcal{O}_{X_{w}}\right]$, while the other expands in terms of Schubert interiors $\xi_{w}=\left[\mathcal{O}_{X_{w}}\left(-\partial X_{w}\right)\right]$. The boundary divisor $\partial X_{w}$ is the union of the Schubert varieties properly contained in $X_{w}$. For positivity in the latter case, it does not suffice to start with the structure sheaf $\mathcal{O}_{Y}$ of a subvariety with rational singularities; only a twist $\mathcal{O}_{Y}(-\partial Y)$ by the ideal sheaf of an effective boundary divisor supporting an ample line bundle will do. The two flavors have nearly identical proofs: the nuanced differences in the statements result from a symmetry between the opposite Schubert variety $X^{w}$ and and arbitrary subvariety $Y$ with rational singularities; see the proof of Theorem 10.4 .

The outline of our method comes from a combination of Anderson's proof [And07] of Graham's equivariant cohomological positivity [Gra01] and Brion's proof of sign alternation for ordinary $K$-theory [Bri02]. First, we translate equivariant statements on $G / P$ into nonequivariant ones on the finite-dimensional approximations of Borel mixing spaces in Section 2.3 and Section 3 . After stating our main results and their previously conjectured corollaries in Sections 4 and 5, we construct the "sufficiently transitive" group action on the mixing space in Section 6. This results in the weak Kleiman transversality principle in Section 77 The difference between the weak version and its strong one is the vanishing result in Section 10, particularly Theorem 10.4 . The proof requires a result on lifting rational singularities under smooth morphisms in Section 8 , along with explicit constructions of such smooth morphisms, based on Bott-Samelson resolutions of singularities, in Section 9

What makes things simpler in cohomology, as opposed to $K$-theory, is that cohomology only requires knowledge on a Zariski dense open subset. Each of the relevant cohomology computations [And07] is carried by an intersection that occurs in one cell of a paving of the mixing space by bundles of affine spaces. As the group action is transitive on each such bundle, ordinary Kleiman transversality suffices. One must then push down to the base of the mixing space, but this operation transfers the positivity to the resulting class.

What fails in $K$-theory? First, unable to restrict to an open cell, we must instead attend to coherent sheaves on closed subvarieties, where the group action is not transitive. Second, pushing forward to the base can have higher direct images, a priori causing mixed negative and positive coefficients.

Getting around the second obstacle is simple, in principle: impose vanishing of the higher direct images. In practice, this is accomplished by stipulating rational singularities, which the Graham-Kumar conjecture [GrKu08, Conjecture 7.1] does explicitly, taking the cue from Brion's phrasing of the result in ordinary $K$-theory [Bri02, Theorem 1]. 
We proceed by suitably modifying Brion's geometric argument that brings KawamataViehweg vanishing to bear.

Dealing with the obstacle of non-transitivity is harder in principle, but it has been made simple in practice by the happy circumstance of recent developments. After Kleiman transversality was generalized to non-transitive group actions in cohomology by Speiser [Spe88], it was recently generalized to homological transversality in $K$-theory for transitive actions by Miller and Speyer [MiSp08]. More recently still, Sierra formulated and proved a $K$-theoretic version for non-transitive group actions [Sie07], and this (Theorem 2.3 is the version we use in the proof of (weak) equivariant homological Kleiman transversality, Theorem 7.2

Looking to the future, it seems the next step would be to combine the equivariant positivity statements here with Mihalcea's in the equivariant quantum setting [Mih06]. In our (non-quantum) setup we can use functoriality to reduce to the full (generalized) flag variety $G / B$; this is one of many things that will have to change to deal with the quantum situation.

\section{Flag varieties, mixing spaces, and $K$-theory}

Write $\mathbb{N} \subset \mathbb{Z} \subset \mathbb{C}$ for the monoid of nonnegative integers, the ring of integers, and the field of complex numbers. All of our schemes are separated and of finite type over $\mathbb{C}$. A variety is assumed to be reduced and equidimensional, but not necessarily irreducible. If a group $G$ acts on $Y$ on the right and on $Z$ on the left, then $Y \times{ }^{G} Z$ is defined to be the quotient of the product $Y \times Z$ by the relation $(y \cdot g, z) \sim(y, g . z)$.

\subsection{Lie theory}

We refer to Borel [Bor91] for the following standard facts and notation. Let $G$ be a complex semisimple algebraic group of adjoint type, and fix a choice $T \subseteq B \subseteq G$ of a maximal torus and Borel subgroup. These have Lie algebras $\mathfrak{t} \subseteq \mathfrak{b} \subseteq \mathfrak{g}$. The weight lattice $\operatorname{Hom}\left(T, \mathbb{C}^{*}\right)$ of $T$ contains the set $R$ of roots. Write $R^{+}$and $R^{-}$for the positive and negative roots, respectively (so $R^{+}$is the set of nonzero weights for the action of $T$ on $\mathfrak{b}$ ). Write $\Delta=\left\{\alpha_{1}, \ldots, \alpha_{n}\right\} \subseteq R^{+}$for the simple roots; thus every positive root $\alpha \in R^{+}$can be written as $\alpha=\sum_{i} k_{i} \alpha_{i}$ with $k_{i} \in \mathbb{N}$.

Since $G$ is adjoint, the root and weight lattices are the same, and $\Delta$ is a basis for the weight lattice. For a weight $\lambda$, write $e^{\lambda}: T \rightarrow \mathbb{C}^{*}$ for the corresponding character.

The normalizer $N(T)$ of the torus $T$ in $G$ has the Weyl group $W=N(T) / T$ as its quotient. (Following a common abuse of notation, we sometimes identify $w \in W$ with a chosen representative in $N(T) \subseteq G$; the choice will never matter.) The simple roots $\alpha_{i}$ determine simple reflections $s_{i} \in W$, and these generate $W$. The length $\ell(w)$ of an element $w \in W$ is the smallest number $\ell$ such that $w$ has an expression $w=s_{i_{1}} \cdots s_{i_{\ell}}$ as a product of simple reflections. When $\ell=\ell(w)$, such an expression is a reduced word for $w$. Let $w_{\circ} \in W$ be the (unique) longest element. The (strong) Bruhat partial order on $W$ is defined by setting $v \leq w$ if $v$ has a reduced word that occurs as a subword (not necessarily consecutive) of a reduced word for $w$. 


\subsection{Flag varieties and Schubert varieties}

The central object of this paper is the quotient $X=G / B$, known as the (complete) flag variety of $G$. Let $B^{-}=w_{\circ} B w_{\circ}$ be the opposite Borel subgroup, so $T=B \cap B^{-}$. The flag variety is paved by the Schubert cells $C_{w}=B w B / B \cong \mathbb{C}^{\ell(w)}$ and also by the opposite Schubert cells $C^{w}=B^{-} w B / B \cong \mathbb{C}^{\operatorname{dim} X-\ell(w)}$ :

$$
X=\coprod_{w \in W} C_{w}=\coprod_{w \in W} C^{w} .
$$

The Schubert varieties $X_{w}$ and opposite Schubert varieties $X^{w}$ are the closures in $X$ of the cells $C_{w}$ and $C^{w}$, respectively. Bruhat order encodes containments among them:

$$
X_{v} \subseteq X_{w} \Leftrightarrow v \leq w \Leftrightarrow X^{v} \supseteq X^{w} .
$$

More generally, if $P \subseteq G$ is a parabolic subgroup, the partial flag variety $G / P$ corresponding to $P$ has a cell decomposition

$$
G / P=\coprod_{w \in W^{P}} C_{w}
$$

where $C_{w}=B w P$ and $W^{P}$ is the set of minimal length representatives for the cosets of $W$ modulo its parabolic subgroup corresponding to $P$. Again write $X_{w}$ and $X^{w}$ for the Schubert and opposite Schubert varieties, the closures of $C_{w}$ and $C^{w}$ in $G / P$.

The Schubert varieties $X_{v}$ and $X^{w}$ intersect properly and generically transversally in the Richardson variety $X_{v}^{w}$. In particular, $X_{v}^{w}$ is empty unless $v \geq w$, and the intersection $X_{w} \cap X^{w}$ is transverse at the point $w B$; moreover, Richardson varieties are irreducible. Schubert varieties are Cohen-Macaulay and have rational singularities [Ram85], and the same is true of Richardson varieties, using [Bri02, Lemmas 1 and 2].

\subsection{Borel mixing spaces and approximations}

We recall some basic notions concerning the Borel mixing space construction; for details, see [Ful07] or [EdGr98]. Let $S$ be an algebraic torus of dimension $r$. Fix a basis $\left\{\beta_{1}, \ldots, \beta_{r}\right\}$ for the weight lattice of $S$, thereby identifying $S$ with $\left(\mathbb{C}^{*}\right)^{r}$. In our applications, $S$ will be a subtorus of the maximal torus $T \subseteq G$ fixed in Section 2.1.

The universal principal $S$-bundle $\mathbb{E} S \rightarrow \mathbb{B} S$ is the union of finite-dimensional algebraic approximations $\mathbb{E}_{m} S \rightarrow \mathbb{B}_{m} S$, which may be constructed as

$$
\mathbb{E}_{m} S=\left(\mathbb{C}^{m+1} \backslash 0\right)^{\times r} \rightarrow\left(\mathbb{P}^{m}\right)^{\times r}=\mathbb{B}_{m} S,
$$

where $S \cong\left(\mathbb{C}^{*}\right)^{r}$ acts on $\left(\mathbb{C}^{m+1} \backslash 0\right)^{\times r}$ diagonally by the standard action. We write

$$
\mathbb{P}=\mathbb{E}_{m} S / S=\mathbb{B}_{m} S
$$

for some fixed sufficiently large $m \gg 0$.

If $Y$ is a scheme with a left $S$-action, the Borel mixing space is $\mathbb{E} S{ }^{S} Y$. As with the universal principal $S$-bundle, we will only use algebraic approximations $\mathcal{Y}=\mathbb{E}_{m} S \times{ }^{S} Y$, 
for some fixed $m \gg 0$. Thus $\mathcal{Y}$ is a Zariski-locally trivial fiber bundle over $\mathbb{P}$ with fiber $Y$. We view the transition $Y \rightsquigarrow \mathcal{Y}$ from an $S$-scheme to its approximate mixing space as a functor on $S$-schemes, and we always indicate it by changing from italic to calligraphic font. When $X=G / P$, we denote by $p$ the projection $\mathcal{X} \rightarrow \mathbb{P}$.

A section $\mathbb{P} \rightarrow \mathcal{Y}$ is equivalent to an $S$-equivariant map $\mathbb{E}_{m} S \rightarrow Y$, as one sees from the following fiber diagram, where the horizontal maps are principal $S$-bundles:

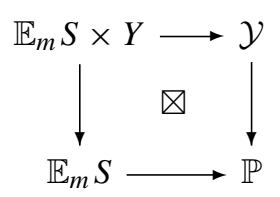

\subsection{Positivity of subtori}

The basis $\left\{\beta_{1}, \ldots, \beta_{r}\right\}$ in Section 2.3 for the weight lattice of the subtorus $S \subseteq T$ is

- positive if the restrictions $\left.\alpha_{1}\right|_{S}, \ldots,\left.\alpha_{n}\right|_{S}$ of the simple roots $\alpha_{1}, \ldots, \alpha_{n}$ are all nonnegative integer combinations of $\beta_{1}, \ldots, \beta_{r}$;

- full if it is positive, and each $\beta_{i}$ equals the restriction of some simple root.

The positivity hypothesis will arise systematically, as it is essential to the geometry in our proof of Theorem 4.2 Notably, it guarantees that the mixing group $\Gamma$ is big enough: positivity begets sections. On the other hand, fullness arises as an essential hypothesis only once in this paper: we mention it in Corollary 5.1. with regard to the diagonal subtorus $S \subseteq T \times T$ inside $G \times G$, so that it can be applied in Corollary 5.2

\subsection{Restrictions and boundary divisors}

For each $j \in\{0, \ldots, m\}$, fix a subspace $\mathbb{P}^{j} \subseteq \mathbb{P}^{m}$. Then, for any $r$-tuple $J=\left(j_{1}, \ldots, j_{r}\right)$ of integers with $0 \leq j_{i} \leq m$, write

$$
\mathbb{P}_{J}=\mathbb{P}^{j_{1}} \times \cdots \times \mathbb{P}^{j_{r}} \subseteq \mathbb{P}
$$

and similarly

$$
\mathbb{P}^{J}=\mathbb{P}^{m-j_{1}} \times \cdots \times \mathbb{P}^{m-j_{r}} \subseteq \mathbb{P} .
$$

Set $|J|=j_{1}+\cdots+j_{r}=\operatorname{dim} \mathbb{P}_{J}=\operatorname{codim} \mathbb{P}^{J}$. The subvariety $\mathbb{P}_{J}$ has boundary divisor

$$
\partial \mathbb{P}_{J}=\mathbb{P}_{\left(j_{1}-1, j_{2}, \ldots, j_{n}\right)} \cup \mathbb{P}_{\left(j_{1}, j_{2}-1, \ldots, j_{n}\right)} \cup \cdots \cup \mathbb{P}_{\left(j_{1}, j_{2}, \ldots, j_{n}-1\right)} .
$$

Our reason for defining $\mathbb{P}_{J}$ is that, for $S$-invariant subschemes $Y \subseteq X=G / B$, we will need to consider the restrictions

$$
\mathcal{Y}_{J}=p^{-1}\left(\mathbb{P}_{J}\right) \cap \mathcal{Y} \subseteq \mathcal{X} \text { and } \mathcal{Y}^{J}=p^{-1}\left(\mathbb{P}^{J}\right) \cap \mathcal{Y} \subseteq \mathcal{X}
$$

of the bundles $\mathcal{Y}$ to $\mathbb{P}_{J}$ and $\mathbb{P}^{J}$. In particular, when $Y=X^{w}$ is an opposite Schubert variety, so $\mathcal{Y}_{J}=\mathcal{X}_{J}^{w}=\left.\mathcal{X}^{w}\right|_{\mathbb{P}_{J}}$, we will additionally need to consider the boundary divisor

$$
\partial \mathcal{X}_{J}^{w}=\left(\left.\mathcal{X}^{w}\right|_{\partial \mathbb{P}_{J}}\right) \cup\left(\bigcup_{v>w} \mathcal{X}_{J}^{v}\right)
$$

This variety is Cohen-Macaulay, by [Bri02, Lemma 4]. 
We will on many occasions need sheaves of the form $\mathcal{O}_{Y}(-\partial Y)$ for which a Weil divisor $\partial Y$ has been defined. When $\partial Y$ is effective, $\mathcal{O}_{Y}(-\partial Y)=\mathcal{I}(\partial Y)$ is the ideal sheaf of $\partial Y$ in $\mathcal{O}_{Y}$. Once $\partial Y$ has been defined, we write $\partial \mathcal{Y}$ for the corresponding Weil divisor on the mixing space. In what follows, we will often write $\mathcal{O}_{Y}(-\partial)$ instead of $\mathcal{O}_{Y}(-\partial Y)$ because our boundary divisors can be notationally complicated varieties. When $\mathcal{O}_{Y}(-\partial Z)$ is written, it serves to emphasize that $Z \neq Y$.

\subsection{Line bundles and canonical sheaves}

A character $\lambda: S \rightarrow \mathbb{C}^{*}$ defines a geometric line bundle

$$
\mathcal{O}(\lambda)=\mathbb{E}_{m} S \times{ }^{S} \mathbb{C}_{\lambda}
$$

on $\mathbb{P}$, where $\mathbb{C}_{\lambda}$ is the one-dimensional representation in which $z \cdot v=\lambda(z) v$ for $v \in \mathbb{C}_{\lambda}$ and $z \in S$. Similarly, $\lambda$ also defines a line bundle

$$
\mathcal{L}_{\lambda}=G \times{ }^{B} \mathbb{C}_{w_{\circ} \lambda}
$$

on $X=G / B$, by extending the character to $B$. The line bundle $\mathcal{L}_{\lambda}$ is to be distinguished from the equivariantly nontrivial but nonequivariantly trivial line bundle $e^{\lambda}=$ $X \times \mathbb{C}_{\lambda}$. Positivity for line bundles defines a partial order on the weight lattice of $S$, in which $\lambda \geq 0$ if and only if $\mathcal{O}(\lambda)$ possesses nonzero global sections; equivalently, $\lambda=c_{1} \beta_{1}+\cdots+c_{r} \beta_{r} \geq 0$ if and only if $c_{i} \geq 0$ for all $i$.

Example 2.1. When the character is $2 \rho=\sum_{\alpha \in R^{+}} \alpha$, the line bundle $\mathcal{L}_{2 \rho}$ is very ample. By considering the simply-connected form of $G$, we find that $\mathcal{L}_{2 \rho}$ has a square root $\mathcal{L}_{\rho}$, which is also a $B$-equivariant very ample line bundle. In general, this line bundle is not equivariant for the adjoint torus, but the bundle $e^{\rho} \mathcal{L}_{\rho}$ is.

For $X=G / B$, we have $\omega_{X} \cong \mathcal{L}_{-2 \rho}, \omega_{X_{w}} \cong e^{-\rho} \mathcal{L}_{-\rho} \otimes \mathcal{O}_{X_{w}}(-\partial)$, and $\omega_{X^{w}} \cong$ $e^{\rho} \mathcal{L}_{-\rho} \otimes \mathcal{O}_{X^{w}}(-\partial)$ as equivariant sheaves [GrKu08, Proposition 2.2(a-b)]. Similarly, for the Bott-Samelson varieties $\widetilde{X}_{w} \stackrel{\varphi}{\rightarrow} X_{w}$, we have $\omega_{\widetilde{X}_{w}} \cong \varphi^{*} e^{-\rho} \mathcal{L}_{-\rho} \otimes \mathcal{O}_{\widetilde{X}_{w}}(-\partial)$ using [Ram85, Proposition 2].

\subsection{Equivariant $K$-theory}

For this subsection, let $X$ be any smooth variety with a left action of the torus $S$. (Shortly, we will return to $X=G / P$ and $S \subseteq T$, a torus in $G$.) Denote by $K_{S}(X)$ the Grothendieck ring of $S$-equivariant vector bundles on $X$. The representation ring is naturally isomorphic to the group algebra

$$
R(S)=\mathbb{Z}[\Lambda]=\bigoplus_{\lambda \in \Lambda} \mathbb{Z} \cdot e^{\lambda}
$$

of the weight lattice $\Lambda=\operatorname{Hom}\left(S, \mathbb{C}^{*}\right)$ of $S$. It coincides with the equivariant Grothendieck ring of a point. Writing $\pi$ for the projection to a point, the pullback $\pi^{*}$ therefore makes $K_{S}(X)$ into an $R(S)$-module. 
Since $X$ is smooth, the natural $R(S)$-module homomorphism $K_{S}(X) \rightarrow K^{S}(X)$ to the Grothendieck group of $S$-equivariant coherent sheaves on $X$ is an isomorphism. The product of the classes of two coherent sheaves $\mathcal{E}$ and $\mathcal{F}$ is the alternating sum

$$
[\mathcal{E}] \cdot[\mathcal{F}]=\sum_{i \geq 0}(-1)^{i}\left[\mathcal{T o r}_{i}^{X}(\mathcal{E}, \mathcal{F})\right]
$$

of their Tor sheaves.

The $K$-homology group $K^{S}$ pushes forward along proper morphisms: $X \stackrel{q}{\rightarrow} Y$ yields

$$
q_{*}[\mathcal{F}]=\sum_{i \geq 0}(-1)^{i}\left[R^{i} q_{*} \mathcal{F}\right],
$$

the point being that all higher direct images are coherent. In particular, if $X$ is smooth and proper, there is an $R(S)$-bilinear pairing on $K_{S}(X)$ given by

$$
\langle[\mathcal{E}],[\mathcal{F}]\rangle_{S}=\pi_{*}([\mathcal{E}] \cdot[\mathcal{F}]),
$$

where $\pi$ is the projection to a point.

\subsection{Equivariant $K$-theory of flag varieties}

Resume the case $X=G / P$ acted on by a torus $S \subseteq T$. Since the subvarieties $X_{w}$ and $X^{w}$ are $S$-stable, their structure sheaves are quotients of $\mathcal{O}_{X}$ by $S$-stable ideal sheaves and hence $S$-equivariant. Let

$$
\mathcal{O}_{w}=\left[\mathcal{O}_{X_{w}}\right] \text { and } \mathcal{O}^{w}=\left[\mathcal{O}_{X^{w}}\right]
$$

be the classes of the structure sheaves of the Schubert varieties and opposite Schubert varieties in $K_{S}(X)$. Because of the cell decompositions in Section 2.2, the sets $\left\{\mathcal{O}_{w}\right\}_{w \in W^{P}}$ and $\left\{\mathcal{O}^{w}\right\}_{w \in W^{P}}$ indexed by the minimal length coset representatives are bases for $K_{S}(X)$ as an $R(S)$-module. Let $\xi^{w}=\left[\mathcal{O}_{X^{w}}(-\partial)\right]$, where $\partial=\partial X^{w}=\bigcup_{v>w} X^{v}$ is the boundary of $X^{w}$, and $\xi_{w}=\left[\mathcal{O}_{X_{w}}(-\partial)\right]$, where $\partial=\partial X_{w}=\bigcup_{v<w} X_{v}$. Then $\left\{\xi^{w}\right\}_{w \in W^{P}}$ and $\left\{\xi_{w}\right\}_{w \in W^{P}}$ are two more bases for $K_{S}(X)$.

Lemma 2.2 ([GrKu08, Proposition 2.1]). The $\mathcal{O}$ and $\xi$ bases of $K_{S}(G / P)$ are dual:

$$
\left\langle\mathcal{O}_{w}, \xi^{v}\right\rangle_{S}=\delta_{w, v} \in R(S) \quad \text { and } \quad\left\langle\mathcal{O}^{w}, \xi_{v}\right\rangle_{S}=\delta_{w, v} \in R(S)
$$

Further basic information and notation concerning the equivariant $K$-theory of flag varieties must wait until Section 3 , where it is shown how the ordinary $K$-theory of mixing spaces approximates it. 


\subsection{Homological transversality}

Our results depend on a certain kind of transversality that simplifies the $K$-theoretic product of two coherent sheaves. This simplification arises separately a couple of times, in the proof of Conjecture 5.3, and in Section 7 as part of the proof of our main result, Theorem 4.1

Two quasicoherent sheaves $\mathcal{E}$ and $\mathcal{F}$ on an arbitrary variety $X$ are homologically transverse if all of their higher Tor sheaves vanish:

$$
\mathcal{T o r}_{j}^{X}(\mathcal{E}, \mathcal{F})=0 \quad \text { for all } j \geq 1
$$

If $\mathcal{E}=\mathcal{O}_{Y}$ is the structure sheaf of a subvariety $Y \subseteq X$, we say that $\mathcal{F}$ is homologically transverse to $Y$. If $X$ is complete and nonsingular, and $Y, Z \subseteq X$ are homologically transverse subvarieties, then in $K(X)$,

$$
\left[\mathcal{O}_{Y}\right] \cdot\left[\mathcal{O}_{Z}\right]=\left[\mathcal{O}_{Y} \otimes \mathcal{O}_{Z}\right]=\left[\mathcal{O}_{Y \cap Z}\right],
$$

where $Y \cap Z$ is the scheme-theoretic intersection. When $Y$ and $Z$ intersect properly (i.e., the sum of their codimensions equals the codimension of every component of their intersection) and both are Cohen-Macaulay, then they are homologically transverse, and their intersection is Cohen-Macaulay; this is the content of [Bri02] Lemma 1].

We shall need the following special case of a theorem due to Sierra [Sie07].

Theorem 2.3. Let $X$ be a variety with a left action of an algebraic group $G$ and let $\mathcal{F}$ be a coherent sheaf on $X$. Suppose that $\mathcal{F}$ is homologically transverse to the closures of the $G$-orbits on $X$. Then for each coherent sheaf $\mathcal{E}$ on $X$ there is a Zariski-dense open set $U \subseteq G$ such that $\mathcal{T o r}_{j}^{X}(\mathcal{E}, g . \mathcal{F})=0$ for all $j \geq 1$ and all $g \in U$.

\subsection{Relative Kawamata-Viehweg vanishing}

Our proof of Theorem 10.4 relies on a relative form of Kawamata-Viehweg vanishing, the relevant version of which we extract from [EsVi92, Corollary 6.11]. In the statement, $f$ nef means $f$-numerically effective: the line bundle $\mathcal{M}$ on $\widetilde{Z}$ has nonnegative intersection with every curve contained in a fiber of $f$. In addition, $f$-big means that the powers of $\mathcal{M}$ give rise to projective morphisms that preserve the dimension of a general fiber of $f$.

Theorem 2.4. Let $f: \widetilde{Z} \rightarrow Z$ be a proper surjective morphism of varieties, with $\widetilde{Z}$ nonsingular. Let $\mathcal{M}$ be a line bundle on $\widetilde{Z}$ such that $\mathcal{M}^{N}(-D)$ is $f$-nef and $f$-big for a normal crossing divisor $D=\sum_{j=1}^{r} a_{j} D_{j}$, where $0<a_{j}<N$ for all $j$. Then

$$
R^{i} f_{*}\left(\mathcal{M} \otimes \omega_{\widetilde{Z}}\right)=0 \quad \text { for all } i>0 .
$$




\section{Approximating equivariant $K$-theory}

Resume the notation from Section 2, including $X=G / P$ and an $r$-dimensional torus $S \subseteq T$ in $G$, along with a (not necessarily positive) basis $\beta_{1}, \ldots, \beta_{r}$ for the weight lattice of $S$. What justifies our omission of the integer $m$ from the notation for approximate Borel mixing spaces in Section 2.3? The essential idea is that, in analogy with the observation by Totaro, Edidin, and Graham [Tot99, EdGr98] underlying equivariant Chow theory, the $K$ theory of the approximate mixing spaces has a well-behaved limit as $m$ increases without bound. This analogy has been precisely formulated by Edidin and Graham themselves in their work on equivariant Riemann-Roch [EdGr00]. For us, the required consequence is as follows.

Proposition 3.1. Let $X=G / P$ and $S \subseteq T$ a torus in $G$. An equation holds in $K_{S}(X)$ if and only if its image holds in $K(\mathcal{X})=K_{S}\left(\mathbb{E}_{m} S \times X\right)$ for some large $m$.

Proof. Let $\widehat{R}(S)_{\mathbb{Q}}$ denote the completion of the rational representation ring $R(S) \otimes_{\mathbb{Z}} \mathbb{Q}$ at its augmentation ideal. Concretely, the augmentation ideal of $R(S) \cong \mathbb{Z}[\Lambda]$ is generated by the elements $1-e^{\lambda}$ for all $\lambda$ in the weight lattice $\Lambda$.

The natural morphism $R(S) \rightarrow \widehat{R}(S)_{\mathbb{Q}}$ is clearly injective. Tensoring this morphism with $K_{S}(X)$ yields the natural map from $K_{S}(X)$ to its completion at the augmentation ideal of $R(S)$ because $K_{S}(X)$ is finitely generated as an $R(S)$-module. Moreover, the morphism remains injective upon this tensoring because $K_{S}(X)$ is flat (in fact, free) as an $R(S)$-module by Lemma 2.2 .

Next, observe that our system $\mathbb{C}^{m \times r}$ of $S$-representations and open subsets $\mathbb{E}_{m} S$ constitute a "good system of representations" in the sense of [EdGr00, Section 2.1]. This "goodness" is easy to verify: it amounts essentially to checking that $S$ acts freely on the open sets, the system is closed under direct sum, and the complements of the open sets are linear subspaces; the details are omitted.

In the presence of goodness, [EdGr00, Theorem 2.1] says the topology on $K_{S}(X)$ coincides with the one induced by the kernels of the surjections $K_{S}\left(\mathbb{C}^{m \times r} \times X\right) \rightarrow$ $K_{S}\left(\mathbb{E}_{m} S \times X\right)$ induced by pullback. The desired result therefore follows from injectivity of the homomorphism to the completion.

Use bars to distinguish classes in the ordinary $K$-ring $K(\mathcal{X})$ of the mixing space from their preimages in $K_{S}(X)$. Thus, we write $\overline{\mathcal{O}}_{w}=\left[\mathcal{O}_{\mathcal{X}_{w}}\right]$ and $\overline{\mathcal{O}}^{w}=\left[\mathcal{O}_{\mathcal{X}} w\right]$ for the usual and opposite Schubert classes, as well as $\bar{\xi}^{w}$ and $\bar{\xi}_{w}$ for their duals (Section 2.8).

Proposition 3.2. $K(\mathcal{X})$ is a $K(\mathbb{P})$-algebra with additive $K(\mathbb{P})$-bases $\left\{\overline{\mathcal{O}}_{w}\right\}_{w \in W^{P}}$ and $\left\{\overline{\mathcal{O}}^{w}\right\}_{w \in W^{P}}$. The dual $K(\mathbb{P})$-bases are $\left\{\bar{\xi}^{w}\right\}_{w \in W^{P}}$ and $\left\{\bar{\xi}_{w}\right\}_{w \in W^{P}}$, respectively.

Proof. The corresponding statement for $K_{S}(X)$ as an algebra over $R(S)$ is a consequence of Lemma 2.2. The desired result follows from the considerations in the proof of Proposition 3.1 $K_{S}\left(\mathbb{E}_{m} S \times X\right)=K(\mathcal{X})$ is the quotient of $K_{S}\left(\mathbb{C}^{m \times r} \times X\right)=K_{S}(X)$ modulo the kernel of the surjective homomorphism $R(S) \rightarrow K(\mathbb{P})$. 
Corollary 3.3. The classes $\left[\mathcal{O}_{\mathbb{P}^{J}}\right]$ are a $\mathbb{Z}$-basis for $K(\mathbb{P})$. Set $\mathcal{O}^{J}=p^{*}\left[\mathcal{O}_{\mathbb{P}^{J}}\right] \in K(\mathcal{X})$. The ordinary $K$-theory $K(\mathcal{X})$ has additive $\mathbb{Z}$-bases

$$
\left\{\mathcal{O}^{J} \cdot \overline{\mathcal{O}}_{w}\right\}_{J, w} \text { and }\left\{\mathcal{O}^{J} \cdot \bar{\xi}_{w}\right\}_{J, w} \text {, where } J \in\{0, \ldots, m\}^{r} \text { and } w \in W^{P} .
$$

Moreover, $\mathcal{O}^{J} \cdot \overline{\mathcal{O}}_{w}=\left[\mathcal{O}_{\mathcal{X}_{w}^{J}}\right]$ and $\mathcal{O}_{J} \cdot \overline{\mathcal{O}}^{w}=\left[\mathcal{O}_{\mathcal{X}_{J}^{w}}\right]$, where $\mathcal{O}_{J}=p^{*}\left[\mathcal{O}_{\mathbb{P}_{J}}\right] \in K(\mathcal{X})$.

The importance of the $K$-classes $\mathcal{O}^{J}$ and $\mathcal{O}_{J}$ on the mixing space is that they provide a geometric interpretation for monomials in the "variables" $1-e^{-\lambda}$.

Lemma 3.4. Let $J \in\{0, \ldots, m\}^{r}$. The ordinary $K$-class $\mathcal{O}^{J} \in K(\mathcal{X})$ is the image of the equivariant "monomial" class $\left(1-e^{-\beta_{1}}\right)^{j_{1}} \cdots\left(1-e^{-\beta_{r}}\right)^{j_{r}} \in K_{S}(X)$.

Proof. Use the exact sequence $0 \rightarrow \mathcal{L}_{-\beta_{i}} \rightarrow \mathcal{O}_{\mathbb{P}} \rightarrow \mathcal{O}_{H^{i}} \rightarrow 0$, where $H^{i}=\mathbb{P}^{\beta_{i}}$ is the component of the boundary $\partial \mathbb{P}$ having $\mathbb{P}^{m-1}$ in the $i^{\text {th }}$ slot. It immediately implies that $\mathcal{O}^{\beta_{i}}=1-e^{-\beta_{i}}$. Clearly $\mathcal{O}^{d \beta_{i}}=\left(1-e^{-\beta_{i}}\right)^{d}$; now use the Künneth formula.

Remark 3.5. Viewing the Chow ring as the associated graded ring of $K$-theory, $1-e^{-\lambda}$ gives rise to the class $\lambda$ (the lowest degree term in the expansion of $1-e^{-\lambda}$ as a power series). This is another indication that $1-e^{-\lambda}$ should be considered "positive".

Since $\mathcal{X}$ is compact, its ordinary $K$-theory has a pairing given by $\langle\alpha, \beta\rangle=\chi(\alpha \cdot \beta)$, where $\chi: K(\mathcal{X}) \rightarrow \mathbb{Z}$ is the Euler characteristic.

Lemma 3.6. Let $I, J \in\{0, \ldots, m\}^{r}$ and $v, w \in W^{P}$. Using (-d) as in Section 2.5.

$$
\left\langle\mathcal{O}^{J} \cdot \overline{\mathcal{O}}_{w}, \mathcal{O}_{I}(-\partial) \cdot \overline{\mathcal{O}}^{v}(-\partial)\right\rangle=\left\langle\mathcal{O}^{J} \cdot \overline{\mathcal{O}}_{w},\left[\mathcal{O}_{\mathcal{X}_{I}^{v}}(-\partial)\right]\right\rangle=\delta_{(J, w),(I, v)} .
$$

Proof. Follow [GrKu08, Prop. 2.1] and [Bri02, Lemma 1]; the details are omitted.

\section{Main theorems}

Theorem 4.1. Let a torus $S \subseteq T$ with a positive basis (Section 2.4P act on $X=G / P$. Fix an $S$-stable subvariety $Y \subseteq X$ and an $S$-stable, Cohen-Macaulay effective divisor $\partial \subset Y$ that supports an ample line bundle on $Y$. Let $\mathcal{Y} \subseteq \mathcal{X}$ be the corresponding mixing spaces, which are fiber bundles over $\mathbb{P}$. Using the bases for $K(\mathcal{X})$ in Corollary 3.3. define $c_{J, w}^{Y}$ and $d_{J, w}^{Y}$ by

$$
\left[\mathcal{O}_{\mathcal{Y}}\right]=\sum_{J, w} c_{J, w}^{Y} \mathcal{O}^{J} \cdot \overline{\mathcal{O}}_{w} \quad \text { and }\left[\mathcal{O}_{\mathcal{Y}}(-\partial)\right]=\sum_{J, w} d_{J, w}^{Y} \mathcal{O}^{J} \cdot \bar{\xi}_{w},
$$

the equations being in $K(\mathcal{X})$. If $Y$ has rational singularities, then

$$
(-1)^{\operatorname{dim} Y-\ell(w)+|J|} c_{J, w}^{Y} \text { and }(-1)^{\operatorname{dim} Y-\ell(w)+|J|} d_{J, w}^{Y}
$$

are nonnegative integers. 
Just as positivity for cohomology is an immediate consequence of Kleiman transversality, Theorem 4.1 is an immediate consequence of the following "positive" homological interpretation of the coefficients $c_{J, w}^{Y}$ and $d_{J, w}^{Y}$ resulting from a generic translation.

Theorem 4.2 (Strong equivariant homological Kleiman transversality). Assume the situation of Theorem 4.1. There is an algebraic mixing group $\Gamma$ acting on $\mathcal{X}$ with finitely many orbits, the closure of each being a mixing space $\mathcal{X}_{w}$ of some Schubert variety $X_{w} \subseteq X$. Fix a general closed point $\gamma \in \Gamma$, and write $\gamma \mathcal{F}$ for the pushforward of any sheaf $\mathcal{F}$ on $\mathcal{X}$ under multiplication by $\gamma \in \Gamma$. If $Y$ has rational singularities, then

$$
(-1)^{\operatorname{dim} Y-\ell(w)+|J|} c_{J, w}^{Y}=\operatorname{dim} H^{\operatorname{dim} Y-\ell(w)+|J|}\left(\mathcal{Y} \cap \gamma \cdot \mathcal{X}_{J}^{w}, \mathcal{O}_{\mathcal{Y} \cap \gamma \cdot \mathcal{X}_{J}^{w}}(-\partial)\right),
$$

where the boundary divisor is $\partial=\partial\left(\mathcal{Y} \cap \gamma \cdot \mathcal{X}_{J}^{w}\right)=\mathcal{Y} \cap \partial\left(\gamma \cdot \mathcal{X}_{J}^{w}\right)$, and

$$
(-1)^{\operatorname{dim} Y-\ell(w)+|J|} d_{J, w}^{Y}=\operatorname{dim} H^{\operatorname{dim} Y-\ell(w)+|J|}\left(\mathcal{Y}_{J} \cap \gamma \cdot \mathcal{X}^{w}, \mathcal{O}_{\mathcal{Y}_{J} \cap \gamma \cdot \mathcal{X}^{w}}\left(-\partial_{\gamma}\right)\right),
$$

where the boundary divisor is $\partial_{\gamma}=\left(\partial \mathcal{Y}_{J}\right) \cap \gamma \cdot \mathcal{X}^{w}$ with $\partial \mathcal{Y}_{J}=\left.\left(\left.\mathcal{Y}\right|_{\partial \mathbb{P}_{J}}\right) \cup(\partial \mathcal{Y})\right|_{\mathbb{P}_{J}}$.

Proof. The group $\Gamma$ is constructed in Section 6, and the statement about its orbits is Lemma 6.3. The construction of $\Gamma$ is where positivity of the basis for the weight lattice of $S$ is crucial, for it guarantees that a certain vector bundle possesses enough sections. Knowing the set of orbit closures allows us easily to express the coefficients $c_{J, w}^{Y}$ and $d_{J, w}^{Y}$ as Euler characteristics in Theorem 7.2, using Sierra's homological transversality (Theorem 2.3 for group actions that are not necessarily transitive. The desired result follows from the more difficult Theorem 10.4 . which says that each Euler characteristic is an alternating sum of terms in which only the last can be nonzero.

Having already explained the roles of Sections 6, 7 and 10 in the proof of Theorem 4.2 , let us complete the discussion by explaining the roles of Sections 8 and 9 . The proof of the vanishing result in Theorem 10.4 is a modification of Brion's proof of the corresponding vanishing for ordinary $K$-theory [Bri02], which is modeled on a Kleiman-type transversality argument. The main difficulty in extending Brion's methods to our situation is the failure of transitivity for our group action on $\mathcal{X}$. It requires us to produce an intermediate result on lifting rational singularities under smooth morphisms in Proposition 8.1. and an explicit construction of such smooth morphisms via Bott-Samelson resolutions of singularities in Proposition 9.2 .

\section{Applications to positivity conjectures}

Corollary 5.1. Fix a positive basis $\beta_{1}, \ldots, \beta_{r}$ for a torus $S \subseteq T$ acting on $X=G / P$ (Section 2.4. For any $S$-stable subvariety $Y \subseteq X$ of $X$ with rational singularities,

$$
\left[\mathcal{O}_{Y}\right]=\sum_{w \in W^{P}} a_{w} \mathcal{O}_{w} \quad \text { with } \quad(-1)^{\operatorname{dim} Y-\ell(w)} a_{w} \in \mathbb{N}\left[e^{-\beta_{i}}-1\right]_{i=1}^{r} \quad \text { for all } w \in W^{P} .
$$

Write $e_{S}^{-\alpha_{i}}$ for the image in $R(S)$ of $e^{-\alpha_{i}} \in R(T)$. If the basis $\beta_{1}, \ldots, \beta_{r}$ is full (Section 2.4 , then positivity for $\left[\mathcal{O}_{Y}\right]$ holds with $\mathbb{N}\left[e_{S}^{-\alpha_{i}}-1\right]_{i=1}^{n}$ in place of $\mathbb{N}\left[e^{-\beta_{i}}-1\right]_{i=1}^{r}$. 
Proof. Apply Proposition 3.1 and Lemma 3.4 to the statement of Theorem 4.1. noting that $\left(e^{-\beta_{i}}-1\right)^{J}=(-1)^{|J|}\left(1-e^{-\beta_{i}}\right)^{J}$. When the basis is full, every monomial in $1-$ $e^{-\beta_{1}}, \ldots, 1-e^{-\beta_{r}}$ is a monomial in $1-e_{S}^{-\alpha_{1}}, \ldots, 1-e_{S}^{-\alpha_{n}}$ by definition.

The previous corollary is a special case of [GrKu08, Conjecture 7.1]. It suffices for the applications to Schubert calculus, such as the following; we do not know if our methods extend to handle the general case, where the subtorus $S$ is arbitrary.

Corollary 5.2 (GrKu08, Conjecture 3.1]). Let $X=G / P$. Using the dual classes $\xi^{w}$ (Section 2.8), the Laurent polynomials $p_{u v}^{w} \in R(T)$ defined by

$$
\xi^{u} \xi^{v}=\sum_{w \in W^{P}} p_{u v}^{w} \xi^{w}
$$

have alternating coefficients when written in terms of the variables $1-e^{-\alpha_{i}}$ :

$$
(-1)^{\ell(w)-\ell(u)-\ell(v)} p_{u v}^{w} \in \mathbb{N}\left[e^{-\alpha_{i}}-1\right]_{i=1}^{n} .
$$

Proof. As Graham and Kumar remark before their Conjecture 7.1, apply Corollary 5.1 to $X \times X$, with $S$ the diagonal subtorus of $T \times T$ and $Y$ the diagonal embedding of $X_{w}$; it is key here that this $S$ possesses a full basis for its weight lattice.

Corollary 5.2 is dual to a positivity conjecture, formulated previously by Griffeth and Ram, for the structure constants with respect to the opposite Schubert class basis. There does not seem to be a direct way to derive one conjecture from the other: the formulas expressing one set of structure constants in terms of the other involve Möbius inversion and are not manifestly positive.

Corollary 5.3 (GrRa04, Conjecture 5.1]). Let $X=G / P$. The Laurent polynomials $c_{u v}^{w} \in R(T)$ defined by

$$
\mathcal{O}^{u} \cdot \mathcal{O}^{v}=\sum_{w \in W^{P}} c_{u v}^{w} \mathcal{O}^{w} \quad \text { for } u, v \in W^{P}
$$

have alternating coefficients when written in terms of the variables $1-e^{-\alpha_{i}}$ :

$$
(-1)^{\ell(w)-\ell(u)-\ell(v)} c_{u v}^{w} \in \mathbb{N}\left[e^{-\alpha_{i}}-1\right]_{i=1}^{n} .
$$

Proof. The coefficient $c_{u v}^{w}$ is the pushforward of the product $\mathcal{O}^{u} \mathcal{O}^{v} \xi_{w} \in K_{T}(X)$ to a point. The equivariant class $\mathcal{O}^{v} \xi_{w}=\left[X_{w}^{v}\left(-X^{v} \cap \partial X_{w}\right)\right]$ is that of a reflexive sheaf on a Richardson variety, with $Y=X_{w}^{v}$ and $\partial=\partial X_{w}$ satisfying the hypotheses of Theorem 4.1. Now apply the results in Section 3 to the statement of Theorem 4.1 (with $S=T$ and $w$ there replaced by $u$ here), noting that $\left(e^{-\alpha_{i}}-1\right)^{J}=(-1)^{|J|}\left(1-e^{-\alpha_{i}}\right)^{J}$.

Remark 5.4. As pointed out in [GrKu08, Proposition 3.13], Corollary 5.3 is equivalent to "signless" positivity for products in the basis of dualizing sheaves: writing

$$
\left[\omega_{X^{u}}\right] \cdot\left[\omega_{X^{v}}\right]=\sum_{w \in W^{P}} d_{u v}^{w}\left[\omega_{X^{w}}\right] \cdot\left[\omega_{G / P}\right],
$$

the Laurent polynomials $d_{u v}^{w} \in R(T)$ satisfy

$$
d_{u v}^{w} \in \mathbb{N}\left[e^{\alpha_{i}}-1\right]_{i=1}^{n} .
$$


Remark 5.5. The positivity results in Corollaries 5.2 and 5.3 hold when restricted to arbitrary subtori $S \subseteq T$, even though we can only show Theorem 4 .1 for subtori with positive bases for their weight lattices. The reason is simply that the statements of the corollaries restrict without obstacle to arbitrary subtori, regardless of the proofs of the corollaries. In particular, sign alternation in ordinary $K$-theory follows from these equivariant results.

\section{A group action on the mixing space}

For the duration of this section, set $X=G / P$, and fix a positive basis $\left\{\beta_{1}, \ldots, \beta_{r}\right\}$ for the weight lattice of a subtorus $S \subseteq T$ (Section 2.4).

The mixing space functor applied to the quotient map $G \rightarrow X$, where $S$ acts on $G$ by left multiplication, expresses the mixing space $\mathcal{X}$ as the quotient of the principal $G$-bundle $\mathcal{G}$ by the action of the parabolic subgroup $P$ on the right.

On the other hand, let $\mathbb{G}=\mathbb{E}_{m} S \times{ }^{S} G$, with $S$ acting on $G$ by inverse conjugation:

$$
t . g=t^{-1} g t \quad \text { for } t \in S \text { and } g \in G .
$$

Since $S$ acts by group automorphisms, $\mathbb{G}$ is a group scheme over $\mathbb{P}$ with fiber $G$. Indeed, the evident multiplication map

$$
\left(\mathbb{E}_{m} S \times G\right) \times \mathbb{E}_{m} S\left(\mathbb{E}_{m} S \times G\right) \rightarrow \mathbb{E}_{m} S \times G
$$

descends to $\mathbb{G} \times \mathbb{P} \mathbb{G} \rightarrow \mathbb{G}$; the inverse map and identity section are defined similarly and satisfy appropriate commutative diagrams. Note, however, that $\mathbb{G}$ is not a principal bundle, since there is no right action of $G$.

Let $B=T U$ be the Levi decomposition of $B$, with $U$ the maximal unipotent group in $B$, and consider the corresponding group scheme

$$
\mathbb{B}=\mathbb{E}_{m} S \times{ }^{S} B \subseteq \mathbb{G}
$$

over $\mathbb{P}$, where again $S$ acts on $B$ by inverse conjugation.

Let $\Gamma_{0}=\operatorname{Hom}(\mathbb{P}, \mathbb{G})$ be the group of global sections of $\mathbb{G}$, i.e., the $\mathbb{P}$-points of this group scheme. Write $\Gamma_{0}(\mathbb{B})=\operatorname{Hom}(\mathbb{P}, \mathbb{B})$; this is a connected algebraic group over $\mathbb{C}$. The following asserts that the group scheme $\mathbb{B}$ is "generated by sections". It requires that the basis $\left\{\beta_{1}, \ldots, \beta_{r}\right\}$ be positive.

Lemma 6.1. For any $x \in \mathbb{P}$ and $p \in \mathbb{B}$ in the fiber over $x$, there is a section $\gamma \in \Gamma_{0}(\mathbb{B})$ such that $p=\gamma(x)$.

Proof. Write $\mathbb{T}=\mathbb{E}_{m} S \times{ }^{S} T$ and $\mathbb{U}=\mathbb{E}_{m} S \times{ }^{S} U$ for the corresponding groups over $\mathbb{P}$. We may assume $p \in \mathbb{T}$ or $p \in \mathbb{U}$.

A section of $\mathbb{T}$ is an $S$-equivariant map $\mathbb{E}_{m} S \rightarrow T$. Since $S$ acts trivially on $T$, this is the same as a map $\mathbb{E}_{m} S / S=\mathbb{P} \rightarrow T$. These are exactly the constant maps, since $\mathbb{P}$ is projective and $T$ is affine, so sections of $\mathbb{T}$ are identified with $T$; in particular, every point of every fiber of $\mathbb{T}$ is in the image of some section. 
Forgetting the group structure, upon fixing a parametrization for each root subgroup $\mathbb{U}$ becomes a vector bundle on $\mathbb{P}$ which splits as a sum of line bundles: $\mathbb{U}=\bigoplus_{\alpha} \mathcal{O}(\alpha)$, where the sum runs over the subset of positive roots that are nontrivial upon restriction to $S$. The positive roots $\alpha \in R^{+}$restrict to nonnegative integer linear combinations of $\beta_{1}, \ldots, \beta_{r}$, by our positivity assumption, and it follows that $\mathbb{U}$ is generated by sections as a vector bundle. The lemma follows from this.

The action of $\left(G L_{m+1}\right)^{r}$ on $\mathbb{P}$ induces a natural action on $\Gamma_{0}(\mathbb{B})$, by precomposition with the projection to $\mathbb{P}$.

Definition 6.2. The mixing group is the semidirect product

$$
\Gamma=\Gamma_{0}(\mathbb{B}) \rtimes\left(G L_{m+1}\right)^{r} .
$$

Thus there is an exact sequence $1 \rightarrow \Gamma_{0}(\mathbb{B}) \rightarrow \Gamma \rightarrow\left(G L_{m+1}\right)^{r} \rightarrow 1$. As a semidirect product of connected groups, $\Gamma$ is also a connected algebraic group.

Lemma 6.3. The mixing group $\Gamma$ acts on the mixing space $\mathcal{X}$ of $X=G / P$ with finitely many orbits, the closure of each orbit being a bundle $\mathcal{X}_{w}$ over $\mathbb{P}$ associated to some Schubert variety $X_{w} \subseteq X$.

Proof. The action of $\Gamma_{0}(\mathbb{B})$ is clear, and $\left(G L_{m+1}\right)^{r}$ acts via its action on $\mathbb{E}_{m} S$ (lifting the action on $\mathbb{P}$ ). Lemma 6.1 implies that the fiber of a $\Gamma$-orbit over a point $p \in \mathbb{P}$ is a $B$-orbit. The result follows from this and the definition of $\mathcal{X}_{w}$.

Remark 6.4. Let $Q \subseteq G$ be the parabolic subgroup generated by $B$ and the centralizer of $S$, so the Levi decomposition of $Q$ is $L U_{Q}$ with $L=C_{G}(S)$ the centralizer of $S$, and $U_{Q}$ the unipotent radical. Let $\mathbb{Q}=\mathbb{E}_{m} S \times{ }^{S} Q$ be the corresponding group scheme over $\mathbb{P}$. Then $\mathbb{B} \subseteq \mathbb{Q} \subseteq \mathbb{G}$, and the above discussion applies with $\mathbb{B}$ replaced by $\mathbb{Q}$, noting that $S$ acts trivially on $L$. The orbits of $\Gamma_{0}(\mathbb{Q}) \rtimes\left(G L_{m+1}\right)^{r}$ are the bundles associated to the $Q$-orbits in $X$.

One can show that $\Gamma_{0}(\mathbb{Q})=\Gamma_{0}$ is the largest group generated by sections in the sense of Lemma 6.1. If the torus $S$ is regular, i.e., $C_{G}(S)=T$, then $Q=B$ and $\mathbb{Q}=\mathbb{B}$.

\section{Generic homological transversality}

This section reduces the computation of the coefficients from Theorem 4.1 to an Euler characteristic using an equivariant homological Kleiman transversality principle in Theorem 7.2. Again let $\mathcal{X}$ be the mixing space of $X=G / B$, with the action of $\Gamma$. By Lemma 6.3, the orbit closures of the $\Gamma$-action are the Schubert bundles $\mathcal{X}_{v}$.

Lemma 7.1. The coherent sheaves $\mathcal{O}_{\mathcal{X}}^{w}$ and $\mathcal{O}_{\mathcal{X}}^{w}(-\partial)$ on $\mathcal{X}$ are homologically transverse to the orbit closures $\mathcal{X}_{v}$ of the $\Gamma$-action on $\mathcal{X}$. 
Proof. Consider the mixing spaces $\mathcal{X}_{J}^{w}$ and $\mathcal{X}_{v}$. These bundles over $\mathbb{P}_{J}$ and $\mathbb{P}$, respectively, intersect in the bundle that is the restriction to $\mathbb{P}_{J}$ of the mixing space $\mathcal{X}_{v}^{w}$ of a Richardson variety. All of these spaces are Cohen-Macaulay, and the intersections are proper, so Section 2.9 applies, and we see that $\mathcal{O}_{\mathcal{X}}^{w}$ is homologically transverse to orbit closures. Similarly, $\partial=\partial \mathcal{X}_{J}^{w}$ is Cohen-Macaulay and intersects $\mathcal{X}_{v}$ properly, so $\mathcal{O}_{\partial}$ is homologically transverse to orbit closures. The claim for $\mathcal{O}_{\mathcal{X}}^{w}(-\partial)$ follows from the exact sequence $0 \rightarrow \mathcal{O}_{\mathcal{X}_{J}^{w}}(-\partial) \rightarrow \mathcal{O}_{\mathcal{X}_{J}^{w}} \rightarrow \mathcal{O}_{\partial} \rightarrow 0$.

Theorem 7.2 (Equivariant homological Kleiman transversality). Fix notation as in Theorems 4.1 and 4.2. but weaken the hypotheses to allow the Cohen-Macaulay subvariety $Y$ not to have rational singularities. Then

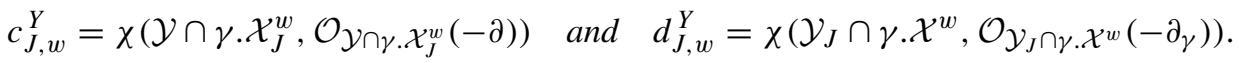

Proof. By Lemma 3.6, we have

$$
\left\langle\left[\mathcal{O}_{\mathcal{Y}}\right],\left[\mathcal{O}_{\mathcal{X}}^{w}(-\partial)\right]\right\rangle=\left\langle\sum_{I, v} c_{I, v}^{Y} \mathcal{O}^{I} \cdot \overline{\mathcal{O}}_{v},\left[\mathcal{O}_{\mathcal{X}_{J}^{w}}(-\partial)\right]\right\rangle=c_{J, w}^{Y}
$$

On the other hand, Theorem 2.3 and Lemma 7.1 guarantee that for general $\gamma \in \Gamma$,

$$
\left[\mathcal{O}_{\mathcal{Y}}\right] \cdot\left[\gamma \mathcal{O}_{\mathcal{X}}^{w}(-\partial)\right]=\left[\mathcal{O}_{\mathcal{Y}} \cap \gamma \cdot \mathcal{X}_{J}^{w}(-\partial)\right]
$$

Since $\Gamma$ is connected, we have

$$
\left\langle\left[\mathcal{O}_{\mathcal{Y}}\right],\left[\mathcal{O}_{\mathcal{X}_{J}^{w}}(-\partial)\right]\right\rangle=\chi\left(\left[\mathcal{O}_{\mathcal{Y}}\right] \cdot\left[\mathcal{O}_{\mathcal{X}_{J}^{w}}(-\partial)\right]\right)=\chi\left(\left[\mathcal{O}_{\mathcal{Y}}\right] \cdot\left[\gamma \mathcal{O}_{\mathcal{X}_{J}^{w}}(-\partial)\right]\right),
$$

and the theorem for $c_{J, w}^{Y}$ follows. The proof for $d_{J, w}^{Y}$ is essentially the same.

\section{Rational singularities}

A desingularization of a variety $X$ is a nonsingular variety $\widetilde{X}$ together with a proper birational map $f: \widetilde{X} \rightarrow X$. As is well known, desingularizations exist for any complex variety $X$. Moreover, if $X$ is equipped with the action of an algebraic group, the desingularization may be chosen so that the action extends to $\widetilde{X}$ and the map $f$ is equivariant. If $D \subseteq X$ is a divisor (invariant for the group action), one can also arrange that $f^{-1} D$ be a normal crossings divisor in $\widetilde{X}$.

If $X$ is a possibly nonreduced scheme, a desingularization of $X$ is a desingularization of the underlying variety $X_{\text {red }}$.

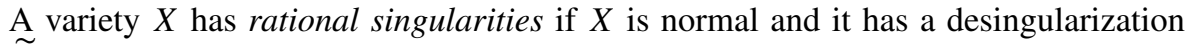
$f: \widetilde{X} \rightarrow X$ such that

$$
\mathbf{R}^{i} f_{*}\left(\mathcal{O}_{\tilde{X}}\right)=0 \quad \text { for all } i>0 .
$$

Equivalently, $X$ has rational singularities if it is Cohen-Macaulay and

$$
f_{*} \omega_{\tilde{X}} \cong \omega_{X}
$$


for a desingularization $f: \widetilde{X} \rightarrow X$. In fact, if either of these conditions holds for some desingularization of $X$, then it holds for all of them.

A morphism of nonsingular varieties $f: X \rightarrow Y$ is smooth if the differential $d f_{x}$ : $T_{x} X \rightarrow T_{f(x)} Y$ is surjective for all $x \in X$. (In differential geometry, this is the same as a submersion.) A smooth morphism is flat (see e.g. [Mum99, III.10, Theorem $3^{\prime}$ ]), and is an open map.

Our proof of the vanishing result in Theorem 10.4 requires the following fact.

Proposition 8.1. Fix a nonsingular complex variety $X$. Let $W$ and $Y$ be varieties with rational singularities, with morphisms $u: W \rightarrow X$ and $v: Y \rightarrow X$. Let $\varphi: \widetilde{W} \rightarrow W$ and $\psi: \widetilde{Y} \rightarrow Y$ be desingularizations. If $\widetilde{W} \rightarrow X$ is a smooth map, then $\widetilde{W} \times_{X} \widetilde{Y} \rightarrow W \times_{X} Y$ is a desingularization and the vanishing in (8.1) holds. If, in addition, $W \times_{X} Y$ is a normal variety, then it has rational singularities.

Proof. Since $\widetilde{W}$ and $\widetilde{Y}$ are nonsingular and $\widetilde{W} \rightarrow X$ is a smooth map, $\widetilde{W} \times{ }_{X} \widetilde{Y}$ is nonsingular. Since $\varphi$ and $\psi$ are proper, so is $\varphi \times \psi: \widetilde{W} \times_{X} \widetilde{Y} \rightarrow W \times_{X} Y$. Birationality follows from that of $\varphi$ and $\psi$, using the openness of $\widetilde{W} \rightarrow X$.

It remains to check condition 8.1 . Since this condition is local on the base, we may assume $X, W$, and $Y$ are affine. Let $f: \widetilde{W} \rightarrow X$ and $g: \widetilde{Y} \rightarrow X$ be the compositions, and let $p: \widetilde{W} \times_{X} \widetilde{Y} \rightarrow \widetilde{W}$ and $q: \widetilde{W} \times_{X} \widetilde{Y} \rightarrow \widetilde{Y}$ be the projections. Since $f: \widetilde{W} \rightarrow X$ is flat, we have $f^{*} \mathbf{R}^{i} g_{*} \mathcal{O}_{\widetilde{Y}} \cong \mathbf{R}^{i} p_{*}\left(q^{*} \mathcal{O}_{\widetilde{Y}}\right)=\mathbf{R}^{i} p_{*}\left(\mathcal{O}_{\widetilde{W} \times X}\right)$, by [Har77] Proposition III.9.3]. Also, writing $g=v \circ \psi$, the Leray spectral sequence shows that $\mathbf{R}^{i} g_{*} \mathcal{O}_{\tilde{Y}}=0$ for $i>0$. (Indeed, for $i>0$ we have $\mathbf{R}^{i} \psi_{*} \mathcal{O}_{\widetilde{Y}}=0$ by rational singularities, and $\mathbf{R}^{i} v_{*} \mathcal{O}_{Y}=0$ since $v$ is an affine map.) It follows that

$$
\mathbf{R}^{i} p_{*}\left(\mathcal{O}_{\widetilde{W} \times X} \tilde{Y}\right)=0 .
$$

Using this and the fact that $W$ has rational singularities, for $i>0$ we have

$$
\mathbf{R}^{i}(\varphi \circ p)_{*}\left(\mathcal{O}_{\widetilde{W} \times_{X} \widetilde{Y}}\right)=0 .
$$

Since $W \times_{X} Y$ and $W$ are affine, [Har77, Proposition III.8.5] gives

$$
\mathbf{R}^{i}(\varphi \times \psi)_{*} \mathcal{O}_{\widetilde{W} \times_{X} \widetilde{Y}}=H^{i}\left(\widetilde{W} \times_{X} \widetilde{Y}, \mathcal{O}_{\widetilde{W} \times_{X} \widetilde{Y}}\right)=\mathbf{R}^{i}(\varphi \circ p)_{*}\left(\mathcal{O}_{\widetilde{W} \times_{X} \widetilde{Y}}\right)=0
$$

for $i>0$, completing the proof that 8.1 holds. The final assertion is an immediate consequence of the definition.

\section{Bott-Samelson varieties}

We will need some basic facts about Bott-Samelson varieties. With the exception of Lemma 9.1 and Proposition 9.2 the following can be found in standard references; see e.g. [Jan87, Chapter 13] or [Mag98].

Let $P_{i}=B s_{i} B \cup B$ be the minimal parabolic subgroup generated by $B$ and $s_{i}$. Let $\underline{w}=\left(s_{i_{1}}, \ldots, s_{i_{r}}\right)$ be a (not necessarily reduced) word in the simple reflections. The corresponding Bott-Samelson variety is

$$
\tilde{X}_{\underline{w}}=P_{i_{1}} \times{ }^{B} \cdots \times{ }^{B} P_{i_{r}} \times{ }^{B}\{p t\}=\left(P_{i_{1}} \times \cdots \times P_{i_{r}}\right) / B^{r},
$$


where $B^{r}$ acts by

$$
\left(b_{1}, b_{2}, \ldots, b_{r}\right) .\left(p_{1}, p_{2}, \ldots, p_{r}\right)=\left(p_{1} b_{1}^{-1}, b_{1} p_{2} b_{2}^{-1}, \ldots, b_{r-1} p_{r} b_{r}^{-1}\right) .
$$

This is a nonsingular variety of dimension $r$, with $B$ acting by left multiplication. It comes with a $B$-equivariant map $\widetilde{X}_{\underline{w}} \rightarrow X=G / B$, sending the class of $\left(p_{1}, \ldots, p_{r}\right)$ to the coset $p_{1} \cdots p_{r} B$; this map has image $X_{w}$, where $w$ is the Demazure product (obtained by using the relations $s_{i}^{2}=s_{i}$ in place of $s_{i}^{2}=1$ ) of the reflections $s_{i_{1}}, \ldots, s_{i_{r}}$.

When $\underline{w}$ is a reduced word for $w$, the map $\widetilde{X}_{\underline{w}} \rightarrow X_{w}$ is a desingularization; if $w \in$ $W^{P}$ is a minimal length coset representative the same is true of the map to $X_{w} \subseteq G / P$. Fix such desingularizations by choosing a reduced word for each $w \in W$, and simply write $\widetilde{X}_{w}$ for the corresponding Bott-Samelson variety.

The desingularization map $\widetilde{X}_{w} \rightarrow X_{w}$ is an isomorphism over the Schubert cell $C_{w}$, identifying $\left\{\left[p_{1}, \ldots, p_{\ell}\right] \in \widetilde{X}_{w} \mid p_{j} \notin B\right.$ for all $\left.j\right\}$ with $C_{w}$. The complement of $C_{w}$ in $\widetilde{X}_{w}$ is the boundary divisor

$$
\partial \widetilde{X}_{w}=\widetilde{X}_{1} \cup \cdots \cup \tilde{X}_{\ell}
$$

where

$$
\tilde{X}_{j}=\left\{\left[p_{1}, \ldots, p_{\ell}\right] \in \tilde{X}_{w} \mid p_{j} \in B\right\} .
$$

Evidently, $\widetilde{X}_{j}$ is isomorphic to the Bott-Samelson variety $\widetilde{X}_{\underline{w}(\widehat{\jmath})}$, where

$$
\underline{w}(\widehat{\jmath})=\left(s_{i_{1}}, \ldots, \widehat{s_{j}}, \ldots, s_{i_{\ell}}\right) \text {; }
$$

in particular, $\partial \widetilde{X}_{w}$ is a transverse union of smooth $B$-stable codimension 1 subvarieties.

Lemma 9.1. The following map is smooth:

$$
B^{-} \times\left(P_{i_{1}} \times \cdots \times P_{i_{r}}\right) / B^{r} \rightarrow G / P, \quad\left(b,\left(p_{1}, \ldots, p_{r}\right)\right) \mapsto b p_{1} \cdots p_{r} P .
$$

Proof. Consider, for $q \geq 1$, the map

$$
B^{-} \times P_{i_{1}} \times \cdots \times P_{i_{q}} \rightarrow G
$$

given by multiplication. When $q=1$ its differential is surjective because of the following: the domain is a homogeneous space for $B^{-} \times P_{i_{1}}$, with action $(b, p) .\left(b^{\prime}, p^{\prime}\right)=$ $\left.\left(b b^{\prime}, p^{\prime} p^{-1}\right)\right)$; the map is equivariant for the natural action of $B^{-} \times P_{i_{1}}$ on the domain and target; and $\operatorname{Lie}(G)=\operatorname{Lie}\left(B^{-}\right)+\operatorname{Lie}\left(P_{i_{1}}\right)$. For $q>1$ we use induction. The map 9.1 can be written as the composition of two multiplication maps

$$
B^{-} \times P_{i_{1}} \times \cdots \times P_{i_{q}} \rightarrow G \times P_{i_{q}} \rightarrow G .
$$

By induction the differential of the first map is surjective at all points of the domain, and the second map obviously has the same property. It follows that 9.1) also has surjective differential everywhere. Upon composing 9.1 with the projection from $G$ to $G / P$ we see that

$$
f: B^{-} \times P_{i_{1}} \times \cdots \times P_{i_{q}} \rightarrow G / P, \quad\left(b,\left(p_{1}, \ldots, p_{q}\right)\right) \mapsto b p_{1} \cdots p_{q} P,
$$


has surjective differential everywhere. On the other hand, $f$ factors through the map $B^{-} \times \widetilde{X}_{w} \rightarrow G / P$, proving our claim.

The opposite Bott-Samelson varieties $\widetilde{X}^{\underline{w}}$ are defined similarly. To be precise, given a word $\underline{w}=\left(s_{i_{1}}, \ldots, s_{i_{r}}\right)$ set

$$
\tilde{X}^{\underline{w}}=P_{i_{1}}^{-} \times{ }^{B^{-}} \cdots \times \times^{B^{-}} P_{i_{r}}^{-} \times{ }^{B^{-}}\{p t\}
$$

where $B^{-}$is the opposite Borel, and the $P_{i}$ are the opposite minimal parabolics. This maps to $X=G / B$ via $\left(p_{i_{1}}, \ldots, p_{i_{r}}\right) \mapsto p_{i_{1}} \cdots p_{i_{r}} w_{\circ} B$. When $\underline{w}$ is a reduced word for $w w_{\circ}, \widetilde{X}^{\underline{w}} \rightarrow X^{w}$ is a resolution of the opposite Schubert variety. As before, fix desingularizations $\widetilde{X}^{w}$ for opposite Schubert varieties by choosing reduced words. Lemma 9.1 applies to opposite Bott-Samelson varieties, exchanging $B$ and $B^{-}$.

Let $\widetilde{\mathcal{X}} \underline{w}$ be the approximate mixing space bundle over $\mathbb{P}$ corresponding to $\widetilde{X}^{\underline{w}}$, and let $\widetilde{\mathcal{X}}_{J}^{w}$ be its restriction to $\mathbb{P}_{J}(\operatorname{Section} 2.5$ ).

Proposition 9.2. The map $\Gamma \times \widetilde{\mathcal{X}}_{J}^{\frac{w}{m}} \stackrel{\widetilde{m}}{\rightarrow} \mathcal{X}$ is smooth.

Proof. Since the map in question is a map of fiber bundles

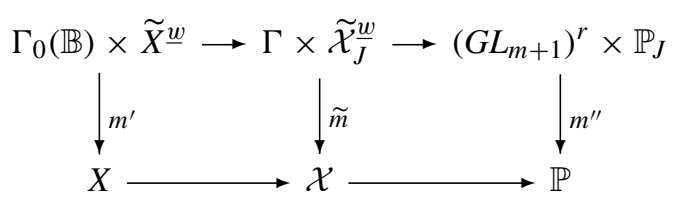

and smoothness is local on the source and on the target, it suffices to prove that $m^{\prime}$ and $m^{\prime \prime}$ are smooth. It is easy to see $m^{\prime \prime}$ is smooth: indeed, $\left(G L_{m+1}\right)^{r}$ acts transitively on $\mathbb{P}$ and $m^{\prime \prime}$ is equivariant, so it is a locally trivial fiber bundle with smooth fiber.

The group $\Gamma_{0}(\mathbb{B})=\operatorname{Hom}(\mathbb{P}, \mathbb{B})$ acts on the fiber over $x \in \mathbb{P}$ by first evaluating at $x$, via a surjective group homomorphism $\Gamma_{0}(\mathbb{B}) \rightarrow B$. Therefore the map $m^{\prime}$ factors through $B \times \widetilde{X}^{\underline{w}} \rightarrow X$, and the latter map is smooth by Lemma 9.1 applied to opposite BottSamelson and Schubert varieties. Since the group homomorphism from $\Gamma_{0}(\mathbb{B}) \rightarrow B$ has surjective differential everywhere, $m^{\prime}$ is also smooth.

Proposition 9.3. The map $\Gamma \times \mathcal{X}^{w} \rightarrow \mathcal{X}$ is flat and has normal fibers.

Proof. As in the proof of Proposition 9.2 it suffices to prove that the action map $B \times$ $X^{w} \stackrel{a}{\rightarrow} X$ is flat and has normal fibers.

We begin with flatness. Note that its image is the union of the Schubert cells $C_{v}$ such that $v \geq w$; this is an open subset $U$ of $X$. Since $U$ is nonsingular and $X^{w}$ is CohenMacaulay, by [Har77, Exercise III.10.9] it suffices to show that the nonempty fibers of $m$ have constant $\operatorname{dimension}$ equal to $\operatorname{dim}\left(B \times X^{w}\right)-\operatorname{dim}(X)$. We will now show that the fibers of $B \times \widetilde{X}^{w} \stackrel{\widetilde{a}}{\rightarrow} X$ map birationally to the fibers of $a$; since $\tilde{a}$ is smooth this completes the proof. Note that the image of the restriction $B \times C^{w} \rightarrow X$ of $a$ contains $C_{v}$ for all $v \geq w$ since $C_{v} \cap C^{w} \neq \varnothing$ if $v \geq w$. Therefore every nonempty fiber of $a$ meets $B \times C^{w}$, and hence is birational to the corresponding fiber of $\tilde{a}$. 
For normality of the fibers, observe that by $B$-equivariance, the fibers of the action map $a$ over the points in $C_{v}$ are all isomorphic to the fiber over $v P \in X$. Write $B_{v}=$ $\operatorname{Stab}_{B}(v P)$ for the stabilizer in $B$ of $v P$, and $U_{v}$ for the subgroup of $B$ generated by those root subgroups not stabilizing $v P$; thus the action map gives an isomorphism $U_{v} \cong C_{v}$. Let $h: C_{v} \rightarrow U_{v}$ be the inverse isomorphism, with $h(u v P)=u$. Then one checks by computing its inverse that the map $B_{v} \times\left(C_{v} \cap X^{w}\right) \rightarrow B \times X^{w}$ given by $(b, x) \mapsto$ $\left(b h(x)^{-1}, x\right)$ is an isomorphism onto the fiber over $v P$, which is therefore normal.

\section{A vanishing theorem for flag bundles}

In this final section, we prove the vanishing theorems required to complete the proof of Theorem 4.1. We need some preliminary results.

Lemma 10.1 ([FuPr98, Lemma, p. 108]). Let $f: \mathcal{W} \rightarrow \mathcal{X}$ be a morphism from a puredimensional scheme $\mathcal{W}$ to a nonsingular variety $\mathcal{X}$, and let $\mathcal{Y}$ be a Cohen-Macaulay closed subscheme of $\mathcal{X}$. Set $Z=f^{-1}(\mathcal{Y})$. If $\mathcal{W}$ is Cohen-Macaulay and $\operatorname{codim}(Z, \mathcal{W})$ $\geq \operatorname{codim}(\mathcal{Y}, \mathcal{X})$, then equality holds and $Z$ is Cohen-Macaulay.

Recall the spaces $\mathcal{X}_{J}^{w}$ from Section 2.5 and the notation from Section 7, and consider the diagram

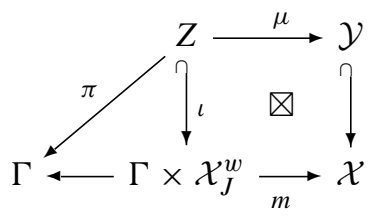

in which $Z$ is defined by the fiber square. Note that

$$
\pi^{-1}(\gamma) \cong \mathcal{Y} \cap \gamma \cdot \mathcal{X}_{J}^{w}
$$

When this fiber is nonempty for general $\gamma \in \Gamma$, it is nonempty for all $\gamma$, so $\pi$ is surjective. We shall assume surjectivity below, since all the statements are trivial if $Z$ is empty.

Lemma 10.2. With notation as above, if $Y$ has rational singularities then $Z$ does, too. In particular, $Z$ is Cohen-Macaulay, so it has a dualizing sheaf $\omega_{Z}$.

Proof. We first construct a desingularization of $Z$. Let $\tilde{Y} \rightarrow Y$ be an $S$-equivariant desingularization of $Y$, and let $\tilde{X}^{w} \rightarrow X_{\tilde{\mathcal{V}}}^{w}$ be the Bott-Samelson desingularization of $X^{w}$, which is also $S$-equivariant. Let $\varphi: \widetilde{\mathcal{Y}} \rightarrow \mathcal{Y}$ and $\psi: \widetilde{\mathcal{X}}_{J}^{w} \rightarrow \mathcal{X}_{J}^{w}$ be the induced desingularizations of bundles, and define notation by the diagram

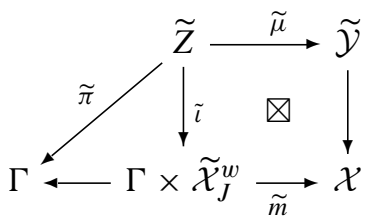


mapping to (10.1). By Proposition 8.1 the map $f: \widetilde{Z} \rightarrow Z$ is a desingularization, and $\mathbf{R}^{i} f_{*} \mathcal{O}_{\widetilde{Z}}=0$ for $i>0$. (The maps to $\Gamma$ do not arise until the proof of Theorem 10.4.) Thus it suffices to show that $Z$ is a normal variety.

In fact, we shall show that $Z$ is Cohen-Macaulay and nonsingular in codimension 1. Since $\widetilde{m}$ is smooth,

$$
\operatorname{dim} \tilde{Z}-\operatorname{dim} \tilde{\mathcal{Y}}=\operatorname{dim}\left(\Gamma \times \tilde{\mathcal{X}}_{J}^{w}\right)-\operatorname{dim} \mathcal{X}
$$

and therefore

$$
\begin{aligned}
\operatorname{codim}\left(Z, \Gamma \times \mathcal{X}_{J}^{w}\right) & =\operatorname{dim}\left(\Gamma \times \tilde{\mathcal{X}}_{J}^{w}\right)-\operatorname{dim} \widetilde{Z}=\operatorname{dim} \mathcal{X}-\operatorname{dim} \tilde{\mathcal{Y}} \\
& =\operatorname{codim}(\mathcal{Y}, \mathcal{X}) .
\end{aligned}
$$

Applying Lemma 10.1 with $\Gamma \times \mathcal{X}_{J}^{w}$ in place of $\mathcal{W}$, we see that $Z$ is Cohen-Macaulay.

To see that $Z$ is nonsingular in codimension 1 , note that the singular locus $\operatorname{Sing}(Z)$ of $Z$ is contained in the union $Z_{1} \cup Z_{2}$, where $Z_{1}=\left(\Gamma \times \operatorname{Sing}\left(\mathcal{X}_{J}^{w}\right)\right) \times \mathcal{X} \mathcal{Y}$ and $Z_{2}=$ $\left(\Gamma \times \mathcal{X}_{J}^{w}\right) \times \mathcal{X} \operatorname{Sing}(\mathcal{Y})$. Indeed, this follows from Propositions 8.1 and 9.2 .

Since $Y$ is normal and the map $\Gamma \times \mathcal{X}_{J}^{w} \rightarrow \mathcal{X}$ is flat, $\operatorname{dim}\left(Z_{2}\right) \leq \operatorname{dim}(Z)-2$. Since Schubert varieties are normal, $\operatorname{Sing}\left(\mathcal{X}_{J}^{w}\right)$ has codimension at least 2 in $\mathcal{X}_{J}^{w}$. Also, $\operatorname{Sing}\left(X^{w}\right)$ is a union of Schubert varieties, so it can be resolved by a union of BottSamelson varieties. Therefore $\Gamma \times \operatorname{Sing}\left(\mathcal{X}_{J}^{w}\right)$ has a resolution $\widetilde{\mathcal{W}} \rightarrow \Gamma \times \operatorname{Sing}\left(\mathcal{X}_{J}^{w}\right)$ such that the composed map $\widetilde{\mathcal{W}} \rightarrow \mathcal{X}$ is smooth, and the dimension argument of the previous paragraph shows that $\operatorname{dim}\left(Z_{1}\right) \leq \operatorname{dim}(Z)-2$.

The proof of Lemma 10.2 also shows the following.

Lemma 10.3. For general $\gamma \in \Gamma$,

$$
\operatorname{dim}\left(\mathcal{Y} \cap \gamma \cdot \mathcal{X}_{J}^{w}\right)=\operatorname{dim} \mathcal{Y}-\operatorname{codim} \mathcal{X}_{J}^{w}=\operatorname{dim} Y-\ell(w)+|J| .
$$

Proof. A general fiber of $\pi: Z \rightarrow \Gamma$ has dimension

$$
\begin{aligned}
\operatorname{dim} Z-\operatorname{dim} \Gamma & =\operatorname{dim} \mathcal{Y}+\operatorname{dim}\left(\Gamma \times \mathcal{X}_{J}^{w}\right)-\operatorname{dim} \mathcal{X}-\operatorname{dim} \Gamma \\
& =\operatorname{dim} \mathcal{Y}+\operatorname{dim} \mathcal{X}_{J}^{w}-\operatorname{dim} \mathcal{X} \\
& =\operatorname{dim} \mathcal{Y}-\operatorname{codim}\left(\mathcal{X}_{J}^{w}, \mathcal{X}\right)
\end{aligned}
$$

as claimed.

Since sheaf cohomology can only be nonzero in cohomological degrees between zero and the dimension of the ambient scheme, the following vanishing theorem finishes the proof of Theorem 4.1. Parts 1 and 2 are, respectively, the statements needed for positivity of the $c$ coefficients and the $d$ coefficients. Part 1 is based on the diagram 10.1 , where $Z$ has a boundary divisor arising from a given boundary on $\Gamma \times \mathcal{X}_{J}^{w}$. Part 2 simply swaps the roles of $\Gamma \times \mathcal{X}^{w}$ and $\mathcal{Y}$ : the boundary divisor on $Z$ is pulled back from the boundary of $\mathcal{Y}_{J}$, which also carries the restriction to $\mathbb{P}_{J}$. 
Theorem 10.4. Assume the hypotheses and notation from Theorems 4.1 and 4.2 including the hypothesis that $Y$ has rational singularities. Fix a general element $\gamma \in \Gamma$.

1. For all $w \in W$ and $i<\operatorname{dim}\left(\mathcal{Y} \cap \gamma \cdot \mathcal{X}_{J}^{w}\right)=\operatorname{dim} Y-\ell(w)+|J|$,

$$
H^{i}\left(\mathcal{Y} \cap \gamma \cdot \mathcal{X}_{J}^{w}, \mathcal{O}(-\partial)\right)=0
$$

Equivalently, for all $w \in W$ and $i>0$,

$$
H^{i}\left(\mathcal{Y} \cap \gamma \cdot \mathcal{X}_{J}^{w}, \omega \mathcal{Y} \cap \gamma \cdot \mathcal{X}_{J}^{w}(\partial)\right)=0 .
$$

2. For all $w \in W$ and $i<\operatorname{dim}\left(\mathcal{Y}_{J} \cap \gamma \cdot \mathcal{X}^{w}\right)=\operatorname{dim} Y-\ell(w)+|J|$,

$$
H^{i}\left(\mathcal{Y}_{J} \cap \gamma \cdot \mathcal{X}^{w}, \mathcal{O}\left(-\partial_{\gamma}\right)\right)=0
$$

Equivalently, for all $w \in W$ and $i>0$,

$$
H^{i}\left(\mathcal{Y}_{J} \cap \gamma \cdot \mathcal{X}^{w}, \omega_{\mathcal{Y}_{J} \cap \gamma \cdot \mathcal{X}^{w}}\left(\partial_{\gamma}\right)\right)=0 .
$$

Proof. The statements beginning "Equivalently" follow from Serre duality, using the fact that $\mathcal{Y} \cap \gamma \cdot \partial \mathcal{X}_{J}^{w}$ and $\partial \mathcal{Y}_{J} \cap \gamma \cdot \mathcal{X}^{w}$ are Cohen-Macaulay to get degeneration of the localto-global spectral sequence (cf. [Bri02, Lemma 4]).

The rest of the proof follows that of [Bri02, Theorem 3]. We will assume $X=G / B$ until the very end of this section; in fact, the entire proof works verbatim for general $G / P$ except the verification of Corollary 10.7 .

Recall the notation defined by the diagram 10.1 . Define the boundary divisor

$$
\partial Z=\mathcal{Y} \times \mathcal{X}\left(\Gamma \times \partial \mathcal{X}_{J}^{w}\right)
$$

of $Z$. For general $\gamma \in \Gamma$, we have

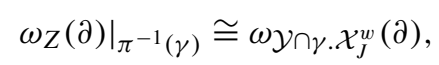

so it will suffice to prove that

$$
R^{i} \pi_{*} \omega_{Z}(\partial)=0 \quad \text { for } i>0 .
$$

We shall accomplish this by applying the Kawamata-Viehweg theorem, in the form of Theorem 2.4, to the desingularization of $Z$ constructed in the proof of Lemma 10.2

Recall the diagram 10.2 . We have seen that $f: \widetilde{Z} \rightarrow Z$ is a desingularization, and $Z$ has rational singularities. Let $\widetilde{\mathcal{X}}_{1}, \ldots, \widetilde{\mathcal{X}}_{\ell}$ be the bundles over $\mathbb{P}$ corresponding to the components of the boundary divisor $\partial \tilde{X}^{w}=\widetilde{X}_{1} \cup \cdots \cup \tilde{X}_{\ell}$.

Lemma 10.5. The boundary divisor

$$
\partial \tilde{\mathcal{X}}_{J}^{w}=\left.\left.\tilde{\mathcal{X}}^{w}\right|_{\partial \mathbb{P}_{J}} \cup \bigcup_{i=1}^{\ell} \widetilde{\mathcal{X}}_{i}\right|_{\mathbb{P}_{J}}
$$

of $\tilde{\mathcal{X}}_{J}^{w}$ supports an ample line bundle on $\tilde{\mathcal{X}}_{J}^{w}$. 
Proof. This follows in a straightforward manner from Example 2.1 by pulling back very ample line bundles. The details are omitted.

The divisor in Lemma 10.5 gives rise to a boundary divisor

$$
\partial \widetilde{Z}=\tilde{\mathcal{Y}} \times \mathcal{X}\left(\Gamma \times \partial \tilde{\mathcal{X}}_{J}^{w}\right)
$$

that is a union of nonsingular irreducible divisors intersecting transversally-that is, with normal crossings - by Proposition 9.2 applied to the components of $\partial \widetilde{\mathcal{X}}_{J}^{w}$ and all of their intersections, each of which is still a Bott-Samelson fibration.

Our next goal is to prove vanishing on $\widetilde{Z}$.

Proposition 10.6. $R^{i} \tilde{\pi}_{*} \omega_{\widetilde{Z}}(\partial)=0$ for $i>0$.

Proof. For this, let $b_{0} \widetilde{\mathcal{X}}_{0}+b_{1} \widetilde{\mathcal{X}}_{1}+\cdots+b_{\ell} \widetilde{\mathcal{X}}_{\ell}$ be the divisor of a very ample line bundle supported on $\partial \widetilde{\mathcal{X}}_{J}^{w}=\bigcup_{i=0}^{\ell} \widetilde{\mathcal{X}}_{i}$, as in Lemma 10.5 , and let $\widetilde{Z}_{i}=\widetilde{\mathcal{Y}} \times \mathcal{X}\left(\Gamma \times \widetilde{\mathcal{X}}_{i}\right)$. Fix an integer $N$ greater $\underset{\widetilde{Z}}{\sim}$ than every $b_{i}$, and write $a_{i}=N-b_{i}$. Set $\mathcal{M}=\mathcal{O}_{\widetilde{Z}}(\partial \widetilde{Z})$ and $D=a_{0} \widetilde{Z}_{0}+\cdots+a_{\ell} \widetilde{Z}_{\ell}$. Then

$$
\mathcal{M}^{\otimes N}(-D)=\mathcal{O}_{\widetilde{Z}}\left(b_{0} \widetilde{Z}_{0}+\cdots+b_{\ell} \widetilde{Z}_{\ell}\right)=\widetilde{\iota}^{*} \mathcal{O}_{\Gamma \times \tilde{\mathcal{X}}_{J}^{w}}\left(\Gamma \times\left(b_{0} \widetilde{\mathcal{X}}_{0}+\cdots+b_{\ell} \widetilde{\mathcal{X}}_{\ell}\right)\right)
$$

is the pullback under the map $\tau$ of a very ample sheaf on $\Gamma \times \mathcal{X}_{J}^{w}$, so it is nef (i.e., its intersection with every curve is nonnegative). In particular, it is $\widetilde{\pi}$-nef and $f$-nef. It is $\tilde{\pi}$-big, because a general fiber $\tilde{\pi}^{-1}(\gamma)=\tilde{\mathcal{Y}} \times \gamma \cdot \widetilde{\mathcal{X}}_{J}^{w}$ maps birationally onto its image under $\tilde{\imath}$. This verifies the hypotheses of Theorem 2.4, whose conclusion says that $R^{i} \widetilde{\pi}_{*}\left(\mathcal{M} \otimes \omega_{\widetilde{Z}}\right)=0$ for $i>0$, concluding the proof of the proposition.

Corollary 10.7. $R^{i} f_{*} \omega_{\widetilde{Z}}(\partial \widetilde{Z})=0$ for $i>0$.

Proof. Continuing notation as in the proof of Proposition 10.6. $\mathcal{M}^{\otimes N}(-D)$ is $f$-nef and $f$-big, the latter because $f$ is birational.

The final constituent in the proof of part 1 is the following.

Proposition 10.8. $f_{*} \omega_{\widetilde{Z}}(\partial \widetilde{Z})=\omega_{Z}(\partial Z)$.

Proof. Consider the factorization of $f: \widetilde{Z} \rightarrow Z$ given by

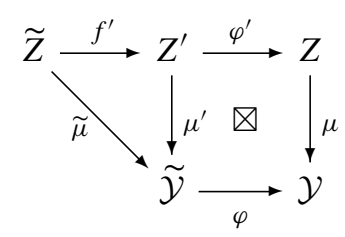

in which the $\otimes$ denotes a fiber square. Note that all fibers of the flat morphism $\mu^{\prime}$ are normal, since they are the same as those of $m: \Gamma \times \mathcal{X}_{J}^{w} \rightarrow \mathcal{X}$, which are normal by Proposition 9.3 Therefore $Z^{\prime}$ is normal by [Mat89, Corollary 23.9]. 
We will establish the following:

$$
\begin{aligned}
& \omega_{\widetilde{Z}}(\partial \widetilde{Z}) \cong \tilde{\mu}^{*}\left(\omega_{\tilde{\mathcal{Y}}} \otimes \varphi^{*} e^{\rho} \mathcal{L}_{\rho}(c \cdot \partial \mathbb{P})\right), \\
& \omega_{Z}(\partial Z) \cong \mu^{*}\left(\omega_{\mathcal{Y}} \otimes e^{\rho} \mathcal{L}_{\rho}(c \cdot \partial \mathbb{P})\right),
\end{aligned}
$$

where $c=\left(c_{1}, \ldots, c_{r}\right)$ is a multi-index, with

$$
c_{i}= \begin{cases}m-j_{i}+1 & \text { if } j_{i}<m, \\ 0 & \text { if } j_{i}=m,\end{cases}
$$

being the coefficient of the corresponding component of $\partial \mathbb{P}$, so that $\omega_{\mathbb{P}^{J}} \cong \mathcal{O}_{\mathbb{P}^{J}}(-c \cdot \partial)$. Granting these isomorphisms for the moment, we have

$$
f_{*}^{\prime} \omega_{\widetilde{Z}}(\partial \widetilde{Z})=f_{*}^{\prime} f^{\prime *} \mu^{\prime *}\left(\omega_{\tilde{\mathcal{Y}}} \otimes \varphi^{*} e^{\rho} \mathcal{L}_{\rho}(c \cdot \partial \mathbb{P})\right)=\mu^{\prime *}\left(\omega_{\tilde{\mathcal{Y}}} \otimes \varphi^{*} e^{\rho} \mathcal{L}_{\rho}(c \cdot \partial \mathbb{P})\right),
$$

using the projection formula and the fact that $f_{*}^{\prime} \mathcal{O}_{\widetilde{Z}}=\mathcal{O}_{Z^{\prime}}$, since $Z^{\prime}$ is normal. Therefore

$$
f_{*} \omega_{\widetilde{Z}}(\partial \widetilde{Z})=\varphi_{*}^{\prime} \mu^{\prime *}\left(\omega_{\tilde{\mathcal{Y}}} \otimes \varphi^{*} e^{\rho} \mathcal{L}_{\rho}(c \cdot \partial \mathbb{P})\right)=\mu^{*} \varphi_{*}\left(\omega_{\tilde{\mathcal{Y}}} \otimes \varphi^{*} e^{\rho} \mathcal{L}_{\rho}(c \cdot \partial \mathbb{P})\right),
$$

because $\mu$ is flat (by Proposition 9.3). Finally, using the projection formula, rational singularities of $\mathcal{Y}$, and (10.6), we obtain

$$
f_{*} \omega_{\widetilde{Z}}(\partial \widetilde{Z})=\mu^{*}\left(\omega_{\mathcal{Y}} \otimes e^{\rho} \mathcal{L}_{\rho}(c \cdot \partial \mathbb{P})\right)=\omega_{Z}(\partial Z),
$$

proving the proposition.

It remains to check 10.5 and 10.6. The morphism $\tilde{\mu}$ is smooth, by 10.2 and Proposition 9.2 Therefore,

$$
\omega_{\widetilde{Z}} \cong \tilde{\mu}^{*} \omega_{\tilde{\mathcal{Y}}} \otimes \omega_{\tilde{Z} / \tilde{\mathcal{Y}}}
$$

Moreover, since the projection $\widetilde{\mathcal{X}}_{J}^{w} \rightarrow \mathbb{P}_{J}$ is a locally trivial fibration, $\omega_{\tilde{\mathcal{X}}_{J}^{w} / \mathbb{P}_{J}}$ is isomorphic to the line bundle on $\tilde{\mathcal{X}}_{J}^{w}$ induced by the equivariant line bundle $\omega_{\widetilde{X}^{w}}$ on the (nonmixing space) Bott-Samelson variety $\tilde{X}^{w}$. The latter bundle is $e^{\rho} \mathcal{L}_{-\rho} \otimes \mathcal{O}_{\tilde{X}^{w}}(-\partial)$, so

$$
\omega \tilde{\mathcal{X}}_{J}^{w} \mathbb{P}_{J} \cong e^{\rho} \mathcal{L}_{-\rho} \otimes \mathcal{O}_{\tilde{\mathcal{X}}_{J}^{w}}\left(-\tilde{\mathcal{X}}_{1}-\cdots-\tilde{\mathcal{X}}_{\ell}\right) \cong e^{\rho} \mathcal{L}_{-\rho} \otimes \mathcal{O}_{\tilde{\mathcal{X}}_{J}^{w}}\left(-\partial \tilde{\mathcal{X}}_{J}^{w}+\partial \mathbb{P}_{J}\right) \text {. }
$$

Finally, using the formula $\omega_{X} \cong \mathcal{L}_{-2 \rho}$ (here we use $X=G / B$ ) and suppressing notation for some obvious pullbacks, we have

$$
\begin{aligned}
& \omega_{\widetilde{Z} / \tilde{\mathcal{Y}}}=\widetilde{\iota}^{*} \omega_{\left(\Gamma \times \tilde{\mathcal{X}}_{J}^{w}\right) / \mathcal{X}} \\
& =\tilde{\iota}^{*}\left(\omega_{\Gamma \times \tilde{\mathcal{X}}_{J}^{w}} \otimes \tilde{m}^{*} \omega_{\mathcal{X}}^{-1}\right) \\
& =\tilde{\iota}^{*}\left(\omega_{\Gamma} \otimes \omega \tilde{\mathcal{X}}_{J}^{w} / \mathbb{P}_{J} \otimes \omega_{\mathbb{P}_{J}} \otimes \tilde{m}^{*} \omega_{\mathcal{X} / \mathbb{P}}^{-1} \otimes \tilde{m}^{*} \omega_{\mathbb{P}}^{-1}\right) \\
& =\tilde{\iota}^{*}\left(\mathcal{O}_{\Gamma} \otimes \tilde{m}^{*} e^{\rho} \mathcal{L}_{-\rho} \otimes \mathcal{O}_{\tilde{\mathcal{X}}_{J}^{w}}\left(-\partial \widetilde{\mathcal{X}}_{J}^{w}+\partial \mathbb{P}_{J}\right) \otimes \mathcal{O}_{\mathbb{P}_{J}}\left(-c^{\prime} \cdot \partial \mathbb{P}_{J}\right) \otimes \tilde{m}^{*} \mathcal{L}_{2 \rho} \otimes \tilde{m}^{*} \mathcal{O}_{\mathbb{P}}\left(c^{\prime \prime} \cdot \partial \mathbb{P}\right)\right) \\
& =\tilde{\iota}^{*}\left(\mathcal{O}_{\Gamma \times \tilde{\mathcal{X}}_{J}^{w}}\left(-\partial \tilde{\mathcal{X}}_{J}^{w}\right) \otimes \tilde{m}^{*} e^{\rho} \mathcal{L}_{\rho} \otimes \tilde{m}^{*} \mathcal{O}_{\mathbb{P}}\left(\left(1_{J}-c^{\prime}+c^{\prime \prime}\right) \cdot \partial \mathbb{P}\right)\right) \\
& =\mathcal{O}_{\widetilde{Z}}(-\partial \widetilde{Z}) \otimes \widetilde{\imath}^{*} \tilde{m}^{*} e^{\rho} \mathcal{L}_{\rho}(c \cdot \partial \mathbb{P}) \\
& =\mathcal{O}_{\widetilde{Z}}(-\partial \widetilde{Z}) \otimes \tilde{\mu}^{*} \varphi^{*} e^{\rho} \mathcal{L}_{\rho}(c \cdot \partial \mathbb{P}) \text {. }
\end{aligned}
$$


Here

$$
c_{i}^{\prime}=\left\{\begin{array}{ll}
j_{i}+1 & \text { if } j_{i}>0, \\
0 & \text { if } j_{i}=0,
\end{array} \quad\left(1_{J}\right)_{i}= \begin{cases}1 & \text { if } j_{i}>0 \\
0 & \text { if } j_{i}=0\end{cases}\right.
$$

and $c^{\prime \prime}=(m+1, \ldots, m+1)$, so $\mathcal{O}_{\mathbb{P}_{J}}\left(-c^{\prime} \cdot \partial \mathbb{P}_{J}\right) \cong \omega_{\mathbb{P}_{J}}$ and $\mathcal{O}_{\mathbb{P}}\left(-c^{\prime \prime} \cdot \partial \mathbb{P}\right) \cong \omega_{\mathbb{P}}$. Thus $1_{J}-c^{\prime}+c^{\prime \prime}=c$.

This proves 10.5. As in Brion's proof, 10.6 is proved similarly, by restricting to the smooth locus of the normal variety $Z$. Thus Proposition 10.8 is proved.

Proposition 10.8 together with Proposition 10.6. Corollary 10.7 and the Leray spectral sequence for $\tilde{\pi}=\pi \circ f$, implies (10.3), which completes the proof of part 1 .

For part 2, the restriction to $\mathbb{P}_{J}$ now appears as $\mathcal{Y}_{J}$ instead of $\mathcal{X}_{J}^{w}$. Using $\otimes$ to denote a pullback square, define notation by the diagram

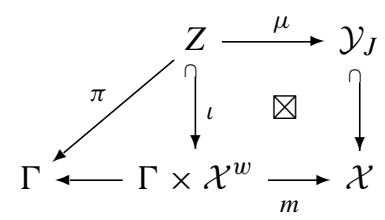

and let $\partial Z=\partial \mathcal{Y}_{J} \times \mathcal{X}\left(\Gamma \times \mathcal{X}^{w}\right)$. Lemma 10.2 holds verbatim in this notation, with the same proof, mutatis mutandis. Similarly, the analogue of Lemma 10.3 still holds. The proof of vanishing for part 2, however, is somewhat different from the proof of part 1 .

Since $\mu$ is flat, we have $\mathcal{O}_{Z}(\partial Z)=\mu^{*} \mathcal{O}_{\mathcal{Y}_{J}}\left(\partial \mathcal{Y}_{J}\right)$. Choose (using [Vil92], say) an $S$ equivariant resolution of singularities $\tilde{Y} \rightarrow Y$ so that $\partial \tilde{\mathcal{Y}}=\varphi^{-1} \partial \mathcal{Y}$ is a normal crossings divisor with ideal sheaf $\mathcal{I}(\partial \mathcal{Y}) \cdot \mathcal{O}_{\tilde{\mathcal{Y}}}$, where $\varphi: \widetilde{\mathcal{Y}} \rightarrow \mathcal{Y}$. The analogue of $[10.2$ has the subscript $J$ on $\tilde{\mathcal{Y}}$ instead of $\tilde{\mathcal{X}}^{w}$; using that notation, the divisor $\partial \widetilde{Z}=\partial \widetilde{\mathcal{Y}}_{J} \times \mathcal{X}\left(\Gamma \times \widetilde{\mathcal{X}}^{w}\right)$ also has normal crossings, and $\mathcal{O}_{\widetilde{Z}}(\partial \widetilde{Z})=\widetilde{\mu}^{*} \mathcal{O}_{\widetilde{\mathcal{Y}}}(\partial \widetilde{\mathcal{Y}})$. Write $\partial \widetilde{Z}=D_{1}+\cdots+D_{\ell}$, with each $D_{i}$ a (nonsingular) irreducible component.

The sheaf $\mathcal{O}_{Z}(\partial)$ is reflexive of rank 1 on the normal variety $Z$. Writing $f: \widetilde{Z} \rightarrow Z$,

$$
\mathcal{H o m}\left(f^{*} \mathcal{O}_{Z}(-\partial), \mathcal{O}_{\widetilde{Z}}\right)=f^{*} \mathcal{O}_{Z}(\partial) / \text { torsion }=f^{*} \mu^{*} \mathcal{O}_{\mathcal{Y}_{J}}(\partial \mathcal{Y}) / \text { torsion }
$$

is a reflexive rank 1 sheaf $\mathcal{M}$ on the smooth variety $\widetilde{Z}$; therefore it is a line bundle. Note that $f_{*} \mathcal{M} \cong \mathcal{O}_{Z}(\partial)$. Since $\tilde{\mu}$ is smooth and $\varphi \tilde{\mu}=\mu f$,

$$
\begin{aligned}
\mathcal{O}_{\widetilde{Z}}(\partial \widetilde{Z}) & =\tilde{\mu}^{*} \mathcal{O}_{\tilde{\mathcal{Y}}_{J}}\left(\varphi^{-1} \partial \mathcal{Y}\right)=\tilde{\mu}^{*}\left(\left(\varphi^{*} \mathcal{O}_{\mathcal{Y}_{J}}(\partial \mathcal{Y})\right) / \text { torsion }\right) \\
& =\left((\varphi \tilde{\mu})^{*} \mathcal{O}_{\mathcal{Y}_{J}}(\partial \mathcal{Y})\right) / \text { torsion }=\mathcal{M}
\end{aligned}
$$

Since $Z$ has rational singularities,

$$
f_{*}\left(\omega_{\widetilde{Z}} \otimes \mathcal{M}\right)=f_{*} \mathcal{H o m}\left(f^{*} \mathcal{O}_{Z}(-\partial), \omega_{\widetilde{Z}}\right)=\omega_{Z}(\partial)
$$

So it will suffice to prove the analogues of Proposition 10.6 and Corollary 10.7

Lemma 10.9. $R^{i} \tilde{\pi}_{*}\left(\omega_{\widetilde{Z}} \otimes \mathcal{M}\right)=0$ for $i>0$, and $R^{i} f_{*}\left(\omega_{\widetilde{Z}} \otimes \mathcal{M}\right)=0$ for $i>0$. 
Proof. It suffices to find a divisor $D$ such that $\mathcal{M}^{\otimes N}(-D)$ is $\tilde{\pi}$-big, $f$-big, $\tilde{\pi}$-nef, and $f$-nef. Fix an ample line bundle $\mathcal{L}$ supported on $\partial \mathcal{Y}$. Writing $\widetilde{\mu}^{*} \varphi^{*} \mathcal{L} \cong \mathcal{O}_{\widetilde{Z}}\left(b_{1} D_{1}+\right.$ $\left.\cdots+b_{\ell} D_{\ell}\right)$, let $a_{i}=N-b_{i}$ for an integer $N$ greater than all the $b_{i}$ 's, and let $D=$ $a_{1} D_{1}+\cdots+a_{\ell} D_{\ell}$. Thus $\mathcal{M}^{\otimes N}(-D) \cong \widetilde{\mu}^{*} \varphi^{*} \mathcal{L}$. This is nef, since it is a pullback of the ample line bundle $\mathcal{L}$; hence it is $\tilde{\pi}$-nef and $f$-nef. It is $\tilde{\pi}$-big and $f$-big for the same reasons as in part 1 . This concludes the proof of the lemma, and with it part 2.

To finish the proof of Theorem 10.4 , it remains to treat the general $G / P$ case, as opposed to the $G / B$ case we have been assuming until now. We proceed as in [Bri02, Lemma 4]. As noted earlier, our entire $G / B$ proof works verbatim for general $G / P$ except for the verification of Corollary 10.7 In particular, the proof for part 2 is the same, so we may assume the situation of part 1 .

For the rest of this proof, write $X=G / P$ and $\widehat{X}=G / B$, and similarly for Schubert varieties and mixing spaces. (Thus we have a proper birational map $\widehat{\mathcal{X}}_{J}^{w} \rightarrow \mathcal{X}_{J}^{w}$ of Schubert varieties, with $w$ a maximal-length coset representative.) Given an $S$-invariant subvariety $Y \subseteq X$, let $\widehat{Y}$ be its inverse image in $\widehat{X}$. Note that the projection $\widehat{X} \rightarrow X$ is a locally trivial fiber bundle, with fiber $P / B$, so the same is true of $\widehat{\mathcal{X}} \rightarrow \mathcal{X}, \widehat{\mathcal{Y}} \rightarrow \mathcal{Y}$, and $\widehat{\mathcal{Y}}_{J} \rightarrow \mathcal{Y}_{J}$. Define notation by the diagram

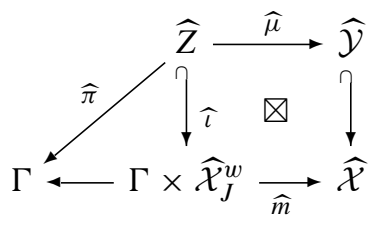

and let $\zeta: \widehat{Z} \rightarrow Z$ be the induced map. It is easy to see that $\zeta$ is proper and birational, and in fact the resolution $f: \widetilde{Z} \rightarrow Z$ factors as $f=\zeta \circ \widehat{f}$, where $\widehat{f}: \widetilde{Z} \rightarrow \widehat{Z}$ is the resolution for the $G / B$ case. Since we know $\widehat{f}_{*} \omega_{\widetilde{Z}}(\partial \widetilde{Z})=\omega_{\widehat{Z}}(\partial \widehat{Z})$, it will suffice to show that

$$
\zeta_{*} \omega_{\widehat{Z}}(\partial \widehat{Z})=\omega_{Z}(\partial Z)
$$

For this, first note that $\zeta_{*} \mathcal{O}_{\widehat{Z}}=\mathcal{O}_{Z}$, since $Z$ is normal, and $\zeta^{-1}(\partial Z)=\partial \widehat{Z}$ from the definitions. Therefore $\zeta_{*} \mathcal{O}_{\widehat{Z}}(-\partial)=\mathcal{O}_{Z}(-\partial)$. Also, we have $\zeta_{*} \omega_{\widehat{Z}}=\omega_{Z}$, since $f$ and $\widehat{f}$ are rational resolutions, so $f_{*} \omega_{\widetilde{Z}}=\omega_{Z}$ and $\widehat{f}_{*} \omega_{\widetilde{Z}}=\omega_{\widehat{Z}}$. Now we compute:

$$
\begin{aligned}
\zeta_{*} \omega_{\widehat{Z}}(\partial \widehat{Z}) & =\zeta_{*}\left(\mathcal{H o m}\left(\mathcal{O}_{\widehat{Z}}(-\partial \widehat{Z}), \omega_{\widehat{Z}}\right)=\mathcal{H o m}\left(\zeta_{*} \mathcal{O}_{\widehat{Z}}(\partial \widehat{Z}), \zeta_{*} \omega_{\widehat{Z}}\right)\right. \\
& =\mathcal{H o m}\left(\mathcal{O}_{Z}(\partial Z), \omega_{Z}\right)=\omega_{Z}(\partial Z) .
\end{aligned}
$$

This proves 10.9), completing the proof of Theorem 10.4

Acknowledgments. The authors are grateful to Michel Brion for his work on $K$-theory of homogeneous spaces [Bri02], which has been indispensable in our investigations; for personally providing additional insight into the subtleties of that work; and for sending helpful comments on a draft of this article. Hearty thanks go to Shrawan Kumar for numerous detailed comments and significant corrections on an earlier draft. We also wish to thank Hiroshi Naruse for valuable corrections, and Susan Sierra for discussions regarding $K$-theory and Cohen-Macaulayness. Substantial parts of this work were completed during two visits by DA to the University of Minnesota. DA was partially funded by NSF graduate and postdoctoral fellowships, DMS-0502170 and DMS-0902967. SG and EM were partially funded by NSF Career Grant DMS-0449102. 


\section{References}

[And07] Anderson, D.: Positivity in the cohomology of flag bundles (after Graham). arXiv:math.AG/0711.0983

[Bor91] Borel, A.: Linear Algebraic Groups. Grad. Texts in Math. 126, Springer (1991) Zbl 0726.20030 MR 1102012

[Bri02] Brion, M.: Positivity in the Grothendieck group of complex flag varieties. J. Algebra 258, 137-159 (2002) Zbl 1052.14054 MR 1958901

[Buc02] Buch, A. S.: A Littlewood-Richardson rule for the $K$-theory of Grassmannians. Acta Math. 189, 37-78 (2002) Zbl 1090.14015 MR 1946917

[EdGr98] Edidin, D., Graham, W.: Equivariant intersection theory. Invent. Math. 131, 595-634 (1998) Zbl 0940.14003 MR 1614555

[EdGr00] Edidin, D., Graham, W.: Riemann-Roch for equivariant Chow groups. Duke Math. J. 102, 567-594 (2000) Zbl 0997.14002 MR 1756110

[Ehr34] Ehresmann, C.: Sur la topologie de certains espaces homogènes. Ann. of Math. (2) 35, 396-443 (1934) Zbl 0009.32903 MR 1503170

[EsVi92] Esnault, H., Viehweg, E.: Lectures on Vanishing Theorems. DMV Seminar 20, Birkhäuser, Basel (1992) Zbl 0779.14003 MR 1193913

[Fu107] Fulton, W.: Equivariant cohomology in algebraic geometry. Lectures at Columbia University; http://www.math.lsa.umich.edu/ dandersn/eilenberg

[FuLa94] Fulton, W., Lascoux, A.: A Pieri formula in the Grothendieck ring of a flag bundle. Duke Math. J. 76, 711-729 (1994) Zbl 0840.14007 MR 1309327

[FuPr98] Fulton, W., Pragacz, P.: Schubert Varieties and Degeneracy Loci. Lecture Notes in Math. 1689, Springer (1998) Zbl 0913.14016 MR 1639468

[Gra01] Graham, W.: Positivity in equivariant Schubert calculus. Duke Math. J. 109, 599-614 (2001) Zbl 1069.14055 MR 1853356

[GrKu08] Graham, W., Kumar, S.: On positivity in $T$-equivariant $K$-theory of flag varieties. Int. Math. Res. Notices 2008, art. ID rnn093, 43 pp. Zbl 1185.14043 MR 2439542

[GrRa04] Griffeth, S., Ram, A.: Affine Hecke algebras and the Schubert calculus. Eur. J. Combin. 25, 1263-1283 (2004) Zbl 1076.14068 MR 2095481

[Har77] Hartshorne, R.: Algebraic Geometry. Grad. Texts in Math. 52, Springer (1977) Zbl 0367.14001 MR 0463157

[Jan87] Jantzen, J. C.: Representations of Algebraic Groups. Pure Appl. Math. 131, Academic Press, Boston (1987) Zbl 0654.20039 MR 0899071

[Kle74] Kleiman, S.: The transversality of a general translate. Compos. Math. 28, 287-297 (1974) Zbl 0288.14014 MR 360616

[Mag98] Magyar, P.: Schubert polynomials and Bott-Samelson varieties. Comment. Math. Helv. 73, 603-636 (1998) Zbl 0951.14036 MR 1639896

[Mat00] Mathieu, O.: Positivity of some intersections in $K_{0}(G / B)$. J. Pure Appl. Algebra 152, 231-243 (2000) Zbl 0978.22016 MR 1783998

[Mat89] Matsumura, H.: Commutative Ring Theory. Cambridge Stud. Adv. Math. 8, Cambridge Univ. Press (1989) Zbl 0666.13002 MR 1011461

[Mih06] Mihalcea, L.: Positivity in equivariant quantum Schubert calculus. Amer. J. Math. 128, 787-803 (2006) Zbl 1099.14047 MR 2230925

[MiSp08] Miller, E., Speyer, D.: A Kleiman-Bertini theorem for sheaf tensor products. J. Algebraic Geom. 17, 335-340 (2008) Zbl 1138.14011 MR 2369089

[Mum99] Mumford, D.: The Red Book of Varieties and Schemes. 2nd ed., Lecture Notes in Math. 1358, Springer (1999) Zbl 0945.14001 MR 1748380 
[PiRa99] Pittie, H., Ram, A.: A Pieri-Chevalley formula in the $K$-theory of a $G / B$-bundle. Electron. Res. Announc. Amer. Math. Soc. 5, 102-107 (1999) Zbl 0947.14025 MR 1701888

[Ram85] Ramanathan, A.: Schubert varieties are arithmetically Cohen-Macaulay. Invent. Math. 80, 283-294 (1985) Zbl 0541.14039 MR 0788411

[Ram87] Ramanathan, A.: Equations defining Schubert varieties and Frobenius splitting of diagonals. Inst. Hautes Études Sci. Publ. Math. 65, 61-90 (1987) Zbl 0634.14035 MR 0908216

[Sie07] Sierra, S.: A general homological Kleiman-Bertini theorem. Algebra Number Theory 3 , 597-609 (2009) Zbl 1180.14048 MR 2578891

[Spe88] Speiser, R.: Transversality theorems for families of maps. In: Algebraic Geometry (Sundance, UT, 1986), Lecture Notes in Math. 1311, Springer, 235-252 (1988) Zbl 0683.14003 MR 0951649

[Tot99] Totaro, B.: The Chow ring of a classifying space. In: Algebraic $K$-theory (Seattle, WA, 1997), Amer. Math. Soc., Providence, RI, 249-281 (1999) Zbl 0967.14005 MR 1743244

[Vi192] Villamayor U., O. E.: Patching local uniformizations. Ann. Sci. École Norm. Sup. (4) 25, 629-677 (1992) Zbl 0782.14009 MR 1198092 